\author{
RESEARCH ARTICLE \\ 10.1029/2019JB017867 \\ Key Points: \\ - HR-EBSD uses cross correlation of \\ diffraction patterns to map lattice \\ distortion \\ - Rotations and strains can be used to \\ calculate GND densities and \\ residual stresses, respectively \\ - Recent developments in data \\ analysis make HR-EBSD suitable for \\ a wide range of rocks
}

Correspondence to:

D. Wallis,

d.wallis@uu.nl

Citation:

Wallis, D., Hansen, L. N., Britton, T. B., \& Wilkinson, A. J. (2019). High-angular resolution electron backscatter diffraction as a new tool for mapping lattice distortion in geological minerals. Journal of Geophysical Research: Solid Earth, 124, 6337-6358. https://doi.org/ 10.1029/2019JB017867

Received 16 APR 2019 Accepted 31 MAY 2019 Accepted article online 3 JUL 2019 Published online 6 JUL 2019

(c)2019. The Authors.

This is an open access article under the terms of the Creative Commons Attribution License, which permits use, distribution and reproduction in any medium, provided the original work is properly cited.

\section{High-Angular Resolution Electron Backscatter Diffraction as a New Tool for Mapping Lattice Distortion in Geological Minerals}

\author{
D. Wallis ${ }^{1}$ (D) L. N. Hansen² ${ }^{(D}$, T. B. Britton ${ }^{3}$ (D) and A. J. Wilkinson ${ }^{4}$ (D) \\ ${ }^{1}$ Department of Earth Sciences, Utrecht University, Utrecht, The Netherlands, ${ }^{2}$ Department of Earth Sciences, University \\ of Oxford, Oxford, UK, ${ }^{3}$ Department of Materials, Imperial College London, London, UK, ${ }^{4}$ Department of Materials, \\ University of Oxford, Oxford, UK
}

Abstract Analysis of distortions of the crystal lattice within individual mineral grains is central to the investigation of microscale processes that control and record tectonic events. These distortions are generally combinations of lattice rotations and elastic strains, but a lack of suitable observational techniques has prevented these components being mapped simultaneously and routinely in earth science laboratories. However, the technique of high-angular resolution electron backscatter diffraction (HR-EBSD) provides the opportunity to simultaneously map lattice rotation and elastic strain gradients with exceptional precision, on the order of $0.01^{\circ}$ for rotations and $10^{-4}$ in strain, using a scanning electron microscope. Importantly, these rotations and lattice strains relate to densities of geometrically necessary dislocations and residual stresses. Recent works have begun to apply and adapt HR-EBSD to geological minerals, highlighting the potential of the technique to provide new insights into the microphysics of rock deformation.

Therefore, the purpose of this review is to provide a summary of the technique, to identify caveats and targets for further development, and to suggest areas where it offers potential for major advances. In particular, HR-EBSD is well suited to characterizing the roles of different dislocation types during crystal plastic deformation and to mapping heterogeneous internal stress fields associated with specific deformation mechanisms/microstructures or changes in temperature, confining pressure, or macroscopic deviatoric stress. These capabilities make HR-EBSD a particularly powerful new technique for analyzing the microstructures of deformed geological materials.

\section{Introduction}

The rates and styles of geodynamic processes on rocky planets emerge from a complex ensemble of underlying processes operating at scales down to the crystal lattices of their constituent minerals. Deciphering this emergent behavior is one of the principal aims of the solid earth geosciences. Analysis of rock microstructures is central to this effort in two respects. First, the microstructures of crystalline materials exert some of the key controls on their mechanical properties. Second, microstructures in deformed materials provide an invaluable record of the processes that operated during, and after, deformation. As such, microstructural data provide a fundamental basis for developing models of deformation behavior and testing their applicability to both experimental and natural systems. Therefore, the development and refinement of techniques that provide microstructural data has been one of the main drivers of recent advances in the fields of experimental rock deformation, structural geology, and tectonics.

A key technique for microstructural analysis of geological materials is electron backscatter diffraction (EBSD, Dingley, 1984; Prior et al., 1999, 2009; Wilkinson \& Hirsch, 1997). EBSD is based on analysis of diffraction patterns acquired in a scanning electron microscope (SEM) and provides a diverse range of microstructural data relevant to geological questions. Some EBSD data sets characterize the microstructures of aggregates of grains. Examples include the distributions of phases, lattice orientations, intergranular misorientations, and grain sizes and shapes (Prior et al., 1999, 2009). Other data sets characterize intragranular microstructures, particularly intragranular lattice misorientations, which are used to constrain the types of dislocations present (Bestmann \& Prior, 2003; Lloyd, 2004; Trimby et al., 1998). Due to the relative ease with which these rich microstructural data can be acquired, EBSD analysis has become routine in many earth science laboratories and, while these data are employed in a wide range of geoscience 
subdisciplines, they have become particularly central to the study of deformed rocks (Ceccato et al., 2018; Cross et al., 2017; Parsons et al., 2016; Prior et al., 2009; Tasaka et al., 2017; Tommasi et al., 2017; Wallis et al., 2018; Weikusat et al., 2017).

Despite its diverse capabilities, conventional EBSD has some key limitations in its ability to characterize subtle intragranular lattice distortions that provide important records of deformation processes. Crystal orientations are most commonly determined by comparing the Hough transforms of diffraction patterns to a database of crystal structures (Adams et al., 1993; Wright \& Adams, 1992). However, locating the peaks in Hough space limits the precision in measurements of (mis)orientation to the order of $\sim 0.1^{\circ}$ (Humphreys et al., 1999). While this angular resolution is sufficient for many purposes, subtle but potentially valuable details of the substructure can be obscured. Furthermore, the precision in misorientation axes decreases with decreasing misorientation angle to the extent that measured misorientation axes can deviate from their true values by tens of degrees for misorientation angles on the order of $1^{\circ}$ (Prior, 1999; Wilkinson, 2001). The presence of geometrically necessary dislocations (GNDs) can result in lattice curvature over a specified length scale, as these dislocations have a net Burgers vector that does not cancel out. Therefore, the precision in measured misorientation angles and axes limits, respectively, the densities and types of GNDs that can be resolved (Wallis et al., 2016). Moreover, the Hough transform-based indexing approach does not allow recovery of information on the variations of elastic strain in the crystal. However, elastic strains and their associated stresses can exert important controls on deformation processes and other microstructural changes.

Developments in the materials sciences over the last decade or so have largely overcome these limitations by developing an alternative data processing approach, termed high-angular resolution electron backscatter diffraction (HR-EBSD). HR-EBSD is based on cross correlation of multiple regions of interest (ROIs) between diffraction patterns to measure the deformation gradient tensor (Britton \& Wilkinson, 2011; Wilkinson, 1996; Wilkinson et al., 2006a). This tensor can be decomposed into lattice rotations and elastic strains, both of which can be measured to a precision of $<10^{-4}$. This precision corresponds to the order of $0.01^{\circ}$ for lattice rotations (Wilkinson, 1996; Wilkinson et al., 2006a). Using this approach, precision in the axes of small misorientations is also dramatically improved over traditional indexing of EBSD patterns (Wilkinson, 2001). The improved precision in misorientation angles and axes translates into improved precision in estimates of the corresponding densities and types of GNDs, respectively (Jiang et al., 2013a; Ruggles, Fullwood, et al., 2016; Wallis et al., 2016). Moreover, the elastic-strain data can be converted into maps of residual stresses retained within the microstructure (Britton \& Wilkinson, 2012a; Jiang et al., 2013b; Karamched \& Wilkinson, 2011).

The ability to precisely map intragranular lattice rotations, GND densities, elastic strains, and residual stresses using EBSD data collected in a standard SEM has led to a wealth of developments in the materials sciences over the past decade. Examples include analyses of distributions of GNDs and elastic strain and residual stress heterogeneity in deformed metals (Jiang, Britton, et al., 2015; Wilkinson \& Randman, 2010), alloys (Britton, Birosca, et al., 2010; Jiang et al., 2016; Littlewood et al., 2011), semiconductors (VilaltaClemente et al., 2017), and ceramics (Villanova et al., 2012), along with characterization of specific processes, such as interactions between dislocations and grain boundaries (Britton \& Wilkinson, 2012a), among many others.

The success of HR-EBSD in the materials sciences alludes to the potential of the technique to offer new insights into the microstructures, deformation processes, and mechanical properties of analogous geological materials. Schäffer et al. (2014) provided an early application of HR-EBSD to geological materials by mapping apparent strains resulting from changes in lattice parameters caused by solid solution in alkali feldspars. Over the past few years, we have undertaken initial HR-EBSD analyses of deformed geological minerals. Examples include mapping GNDs and residual stress heterogeneity in single crystals of olivine (Kumamoto et al., 2017; Wallis et al., 2016; Wallis, Hansen, et al., 2017) and mapping GNDs in aggregates of olivine (Boneh et al., 2017; Kumamoto et al., 2017; Qi et al., 2018) and quartz (Wallis, Parsons, et al., 2017). These examples demonstrate the great potential of the technique and highlight some subtle but important considerations for analysis of geological materials in particular. Therefore, it is timely to provide a review of the application of HR-EBSD in the earth sciences. We begin by providing summaries of the technique and practical aspects of its application and then highlight key points by providing illustrative 


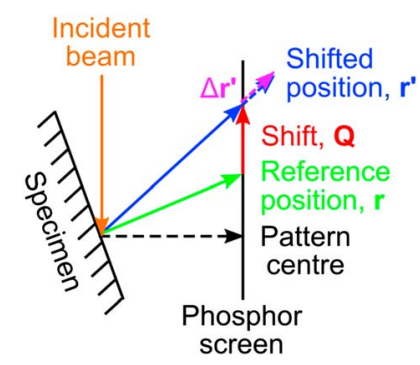

Figure 1. Schematic of the geometry involved in describing a shift, $\mathbf{Q}$, of a point within a diffraction pattern from a reference position, $\mathbf{r}$, to a shifted position, $\mathbf{r}^{\prime}$. The component of $\mathbf{r}^{\prime}$ extending out of the plane of the phosphor screen, $\Delta \mathbf{r}^{\prime}$, cannot be directly detected from a shift within the plane of the phosphor screen (after Britton \& Wilkinson, 2012b). examples. We finish by discussing the strengths and limitations of the technique and summarizing potential research directions.

\section{Technique Development}

HR-EBSD has developed gradually over the past 25 years, but only recently have developments made it suitable for wide-ranging application to the variety of typical rock microstructures. Early works recognized the potential of measuring small shifts of features within EBSD patterns to reveal small lattice rotations and elastic strains (Troost et al., 1993; Wilkinson, 1996, 2000, 2001). A major development was made by Wilkinson et al. (2006a), who presented a practical and mathematical framework for estimating 8 degrees of freedom in the displacement gradient tensor, describing rotations and strains, from diffraction patterns obtained on megapixel charge-coupled device detectors. The final degree of freedom relates to the hydrostatic strain, which cannot be measured directly but can be determined by constraining the surface normal stress to zero (Wilkinson et al., 2006b). Subsequent methodological refinements have focused on assessing and improving the accuracy and precision of strain measurement and extending the potential applications to more challenging microstructures (Britton, Maurice, et al., 2010; Britton \& Wilkinson, 2011, 2012b; Britton et al., 2013a, 2013b; Maurice \& Fortunier, 2008; Maurice et al., 2012; Plancher et al., 2015; Tong et al., 2015; Villert et al., 2009; Wilkinson et al., 2014). A recent advance that is particularly important for analysis of geological materials, which are commonly deformed to large plastic strains, has been the development of routines for mapping elastic strains in the presence of lattice rotations of several degrees, such as subgrain boundaries (Britton \& Wilkinson, 2011, 2012b; Maurice et al., 2012). Below, we summarize the key elements of the technique that are necessary to appreciate its application to geological materials.

\section{Principles of HR-EBSD}

\subsection{Measuring Rotations, Elastic Strains, and Residual Stresses}

HR-EBSD data are derived from mapping small distortions within stored images of diffraction patterns. In this section, we summarize the method of mapping these distortions based on the work of Wilkinson et al. (2006a) and improved by Britton and Wilkinson (2011, 2012b). For HR-EBSD, the Hough transform is used only to determine the orientation of a single reference point in each grain. Consequently, the accuracy of absolute crystal orientations in HR-EBSD data sets is the same as the original EBSD data, on the order of a few degrees and limited by specimen alignment and geometric distortions (Nolze, 2007). However, HREBSD processing improves the precision in relative orientations, that is, misorientations, between a reference point in a grain of interest and all other points in that grain. An array of small, typically $256 \times 256$ pixel, ROIs are extracted from the same positions within each $\sim 1,000 \times 1,000$ pixel diffraction pattern. A minimum of four dispersed ROIs are required from each diffraction pattern, but typically 20 or more are employed to overdetermine the deformation gradient tensor. Misorientations between the reference point and all other points in the grain are determined by cross correlating each ROI from each diffraction pattern with the corresponding ROI from the reference pattern. For computational speed, the fast Fourier transforms of the ROI are computed, and the cross correlation is performed in Fourier space. This procedure has the added benefit that band-pass filters can be easily applied to reduce high-frequency noise and long-wavelength intensity gradients within the ROIs. The position of the peak in the cross-correlation function gives the translation that best aligns the ROIs from the reference and test patterns, that is, it determines how the position of the ROI is shifted between one pattern and the other. To improve the precision in the shift measurement, the peak in the cross-correlation function is interpolated to estimate its position to \pm 0.05 pixels, which provides a precision in strain of $10^{-4}$ when working with megapixel diffraction patterns (Villert et al., 2009).

The geometry of a displaced ROI in a diffraction pattern is illustrated schematically in Figure 1 . The position of the center of a ROI in the reference pattern is given by vector $\mathbf{r}$. In a test pattern, the feature found in the reference pattern at $\mathbf{r}$ would be instead projected to position $\mathbf{r}^{\prime}$. Only the component of this displacement that lies within the plane of the phosphor screen is detected as a shift, $\mathbf{Q}$, in the position of the ROI. The component of $\mathbf{r}^{\prime}$ extending out of the plane of the phosphor screen, $\Delta \mathbf{r}^{\prime}$, is not detected. 


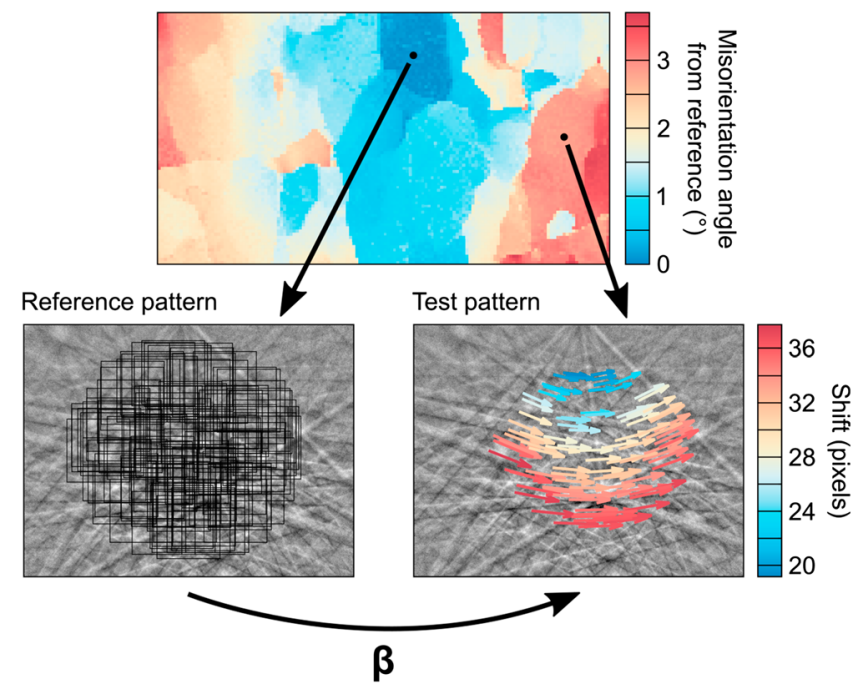

Figure 2. Example of shifts in regions of interest between the reference pattern and a test pattern in a different subgrain in quartz (sample P12/058 of Parsons et al., 2016; Wallis, Parsons, et al., 2017). One hundred regions of interest, each $256 \times 256$ pixels, are marked by black boxes on the reference pattern. Exaggerated shifts in these regions of interest are indicated by arrows on the test pattern. The true magnitude of the shifts is indicated by the color scale. The shifts in the regions of interest are described by the deformation gradient tensor, $\beta$.
The mapping of $\mathbf{r}$ to $\mathbf{r}^{\prime}$ is described by

$$
\mathbf{r}^{\prime}=\beta \mathbf{r},
$$

where $\beta$ is the deformation gradient tensor. An example of this mapping is presented in Figure 2. $\beta$ is determined by fitting the shifts in the ROIs. When the elastic strains and lattice rotations are small, $\beta$ can be additively decomposed as

$$
\beta=\varepsilon+\omega+\mathbf{I},
$$

where $\mathbf{I}$ is the identity matrix and $\varepsilon$ and $\boldsymbol{\omega}$ are the infinitesimal strains and rotations, respectively, given by

$$
\varepsilon=\frac{1}{2}\left(\beta+\beta^{\mathrm{T}}\right)-\mathbf{I}
$$

where $^{\mathbf{T}}$ indicates the transpose, and

$$
\omega=\frac{1}{2}\left(\beta-\beta^{\mathrm{T}}\right)-\mathbf{I}
$$

The stress normal to the section is assumed to be zero, and this provides constraint to solve for the hydrostatic strain.

The cross-correlation basis of HR-EBSD places limits on the maximum misorientation angle between pairs of patterns that can be analyzed. The method originally presented by Wilkinson et al. (2006a) can measure lattice rotations up to misorientation angles of approximately $8^{\circ}$, beyond which the patterns become too dissimilar and distorted for accurate measurements (Britton \& Wilkinson, 2011). Elastic strain measurements are limited to an even more restricted range of misorientation angles of approximately $1^{\circ}$, beyond which the large shifts due to the rotations swamp the much smaller signal from elastic strains (Britton \& Wilkinson, 2012b). Beyond this angular range, errors introduced to the strain estimate by the large rotations commonly result in spuriously large phantom strains (Britton \& Wilkinson, 2012b).

To extend the range of misorientation angles over which rotations and strains can be measured, Britton and Wilkinson (2012b) proposed a more advanced three-step approach. First, an initial pass of cross correlation is employed to obtain an infinitesimal rotation matrix based on the procedure outlined above, while elastic strains are initially ignored. Second, the infinitesimal rotations are used to estimate a finite rotation matrix, $\boldsymbol{\Omega}$, by

$$
\mathbf{\Omega}=\left(\begin{array}{ccc}
\cos \omega_{12} & \sin \omega_{12} & 0 \\
-\sin \omega_{12} & \cos \omega_{12} & 0 \\
0 & 0 & 1
\end{array}\right) \times\left(\begin{array}{ccc}
1 & 0 & 0 \\
0 & \cos \omega_{23} & \sin \omega_{23} \\
0 & -\sin \omega_{23} & \cos \omega_{23}
\end{array}\right) \times\left(\begin{array}{ccc}
\cos \omega_{31} & 0 & -\sin \omega_{31} \\
0 & 1 & 0 \\
\sin \omega_{31} & 0 & \cos \omega_{31}
\end{array}\right)
$$

This finite rotation matrix is used to make a virtual rotation of the test pattern back into an orientation similar to that of the reference pattern. In this step, the intensities in the measured test pattern are interpolated and remapped to generate a rotated test pattern. Third, a second pass of cross correlation is employed to measure elastic strains from the rotated test pattern (which is now much more similar to the reference pattern), along with any small corrections to the rotations. A similar methodology has also been presented by Maurice et al. (2012). This remapping approach extends the angular range over which both rotations and strains can be measured to $\sim 11^{\circ}$. This procedure dramatically extends the range of materials in which elastic strains can be measured and, importantly, allows elastic strain measurements in materials containing large plastic strain gradients manifesting as intragranular misorientations of several degrees. Based on the elastic strain measurements, it is straightforward to calculate the corresponding residual stresses based on Hooke's law and the elastic stiffness tensor of the material (Britton \& Wilkinson, 2012a; Karamched \& Wilkinson, 2011). 
As HR-EBSD data sets are typically maps containing large numbers of individual measurements it has been important to establish some data quality metrics to assure that points with poorer quality data (perhaps associated with poor condition of the specimen surface) can be readily identified and used to exclude such points from further analysis. Two main measures are used (Britton \& Wilkinson, 2011). The first metric is the geometric mean of the cross-correlation peak heights determined for each region of interest. If the pattern matching and registration has worked well, values close to unity are obtained, but the value falls if, for example, the pattern is from a different grain or if dust or a surface pore lead to partial shadowing of the pattern. The second metric is the (weighted) mean angular error, which assesses the fit of the solution for the deformation gradient tensor, $\beta$, to the measured shifts in the ROIs. The mean angular error is the arithmetic mean of the difference in angular shift predicted at the center of each ROI and that actually measured. Therefore, strain and rotation measurements that are smaller than the mean angular error should be treated with caution.

\subsection{Estimating Densities and Types of GNDs}

Curvature of the crystal lattice results from the presence of GNDs. Analysis of GNDs can be conducted through the dislocation density tensor, $\alpha$, using Nye-Kröner analysis (Kröner, 1958; Nye, 1953). The presence of dislocations introduces spatial gradients, in the directions $x_{i}$, of lattice orientation (measured as rotations) and elastic strain, which contribute to the components $\alpha_{i j}$ of $\alpha$ by

$$
\alpha_{i j}=\left[\begin{array}{ccc}
\frac{\partial \omega_{12}}{\partial x_{3}}-\frac{\partial \omega_{31}}{\partial x_{2}} & \frac{\partial \omega_{13}}{\partial x_{1}} & \frac{\partial \omega_{21}}{\partial x_{1}} \\
\frac{\partial \omega_{32}}{\partial x_{2}} & \frac{\partial \omega_{23}}{\partial x_{1}}-\frac{\partial \omega_{21}}{\partial x_{3}} & \frac{\partial \omega_{21}}{\partial x_{2}} \\
\frac{\partial \omega_{32}}{\partial x_{3}} & \frac{\partial \omega_{13}}{\partial x_{3}} & \frac{\partial \omega_{31}}{\partial x_{2}}-\frac{\partial \omega_{32}}{\partial x_{1}}
\end{array}\right]+\left[\begin{array}{cccc}
\frac{\partial \varepsilon_{12}}{\partial x_{3}}-\frac{\partial \varepsilon_{13}}{\partial x_{2}} & \frac{\partial \varepsilon_{13}}{\partial x_{1}}-\frac{\partial \varepsilon_{11}}{\partial x_{3}} & \frac{\partial \varepsilon_{11}}{\partial x_{2}}-\frac{\partial \varepsilon_{12}}{\partial x_{1}} \\
\frac{\partial \varepsilon_{22}}{\partial x_{3}}-\frac{\partial \varepsilon_{23}}{\partial x_{2}} & \frac{\partial \varepsilon_{23}}{\partial x_{1}}-\frac{\partial \varepsilon_{21}}{\partial x_{1}} & \frac{\partial \varepsilon_{21}}{\partial x_{2}}-\frac{\partial \varepsilon_{22}}{\partial x_{1}} \\
\frac{\partial \varepsilon_{32}}{\partial x_{3}}-\frac{\partial \varepsilon_{33}}{\partial x_{2}} & \frac{\partial \varepsilon_{33}}{\partial x_{1}}-\frac{\partial \varepsilon_{31}}{\partial x_{3}} & \frac{\partial \varepsilon_{31}}{\partial x_{2}}-\frac{\partial \varepsilon_{32}}{\partial x_{1}}
\end{array}\right] .
$$

The components of $\alpha_{i j}$ relate to the densities, $\rho^{\mathrm{s}}$, of $s_{\max }$ different types of dislocation, with Burgers vectors $\mathbf{b}^{s}$ and line directions $\mathbf{1}^{s}$, through

$$
\alpha_{i j}=\sum_{s=1}^{s_{\max }} \rho^{s} b_{i}^{s} l_{j}^{s}
$$

It is clear from equations (6) and (7) that different types of dislocation generate different rotation and strain gradients and hence contribute differently to the components of $\alpha_{i j}$. For instance, screw dislocations (with Burgers vectors parallel to their line directions) contribute to the diagonal components, whereas edge dislocations (with Burgers vectors perpendicular to their line directions) contribute to the off-diagonal components. Dislocations of mixed character are represented by densities of their end-member edge and screw components. Rotation and strain gradients in the direction normal to the specimen surface (i.e., in the $x_{3}$ direction) cannot be measured from a two-dimensional EBSD map. The absence of this information leaves only the $\alpha_{i 3}$ terms fully determined. However, often the rotation gradients are larger than the elastic strain gradients (an assessment that can be made from HR-EBSD data but not from conventional EBSD data), in which case, the elastic strain gradients can be neglected entirely or only the measurable terms included in the analysis (Wilkinson \& Randman, 2010). In this case, five components of $\alpha_{i j}\left(\alpha_{12}, \alpha_{13}, \alpha_{21}, \alpha_{23}\right.$, and $\left.\alpha_{33}\right)$ can be determined directly, along with the difference between two of the remaining components, that is, $\alpha_{11}-\alpha_{22}$ (Pantleon, 2008).

For simple cubic crystals with $\mathbf{b}^{s}$ and $\mathbf{l}^{s}$ of nine (or fewer) dislocation types constrained to lie along the cube axes, as originally considered by Nye (1953), equation (7) provides an intuitive and unambiguous relationship between the lattice curvature and dislocation content (Arsenlis \& Parks, 1999; Sun et al., 2000; Wilkinson \& Randman, 2010). However, to analyze more complex crystal structures with more numerous possible dislocation types, a more general approach is required. The problem of estimating the densities of each type of GND from the available components of $\alpha_{i j}$ can be set out as

$$
\mathbf{A} \rho=\lambda,
$$

where $\rho$ is a vector of the densities of the $s_{\max }$ dislocation types and $\lambda$ is a vector containing the measurable components of lattice curvature, of which there are six, corresponding to components of $\alpha_{i j}$ (Pantleon, 2008). 
A is a $6 \times s_{\max }$ matrix in which each column contains the dyadic of the Burgers vector and unit line direction of the sth dislocation type (Arsenlis \& Parks, 1999; Britton \& Wilkinson, 2012a). Equation (8) can be solved using the right Moore-Penrose inverse,

$$
\rho=\mathbf{A}^{\mathrm{T}}\left(\mathbf{A A}^{\mathrm{T}}\right)^{-\mathbf{1}} \lambda
$$

(Arsenlis \& Parks, 1999; Wilkinson \& Randman, 2010). Equation (9) can be used to directly calculate the best fit values of $\rho$ since it inherently solves equation (8) in a least squares sense by minimizing the $L_{2}$-norm of the dislocation densities,

$$
L_{2}=\left[\sum_{s=1}^{s_{\max }}\left[\rho^{s}\right]^{2}\right]^{1 / 2}
$$

(Dunne et al., 2012). This approach yields a unique solution for crystal structures in which the analysis can reasonably be limited to consideration of six dislocation types or fewer and has been employed to estimate GND densities in olivine deformed at high temperature (Boneh et al., 2017; Kumamoto et al., 2017; Qi et al., 2018; Wallis et al., 2016; Wallis, Hansen, et al., 2017). However, for crystal structures with more than six dislocation types, there are typically many combinations of dislocation types and densities that are geometrically capable of generating measured lattice curvature, and the problem of solving equation (8) is underconstrained (i.e., A has more columns than rows). In such cases, an additional constraint must be employed to select an optimal solution. One such approach that has been applied to cubic and hexagonal metals (Britton \& Wilkinson, 2012a; Jiang et al., 2013c; Wilkinson \& Randman, 2010) is to weight the dislocation densities by their line energy, $E^{s}$, in the minimization of $L_{1}$ in

$$
L_{1}=\sum_{s=1}^{s_{\max }}\left|\rho^{s} E^{s}\right|
$$

The energies of edge and screw dislocations, $E_{\text {edge }}$ and $E_{\text {screw }}$, respectively, used in the $L_{1}$ minimization scheme are in the ratio

$$
\frac{E_{\text {edge }}}{E_{\text {screw }}}=\frac{1}{1-v}
$$

where $v$ is the Poisson's ratio (Wilkinson \& Randman, 2010). This approach has been applied to quartz, considering 19 dislocation types grouped into six families (Wallis, Parsons, et al., 2017). As calculation of the energies of individual dislocations and the combined energies of groups of dislocations is complex (Mesarovic et al., 2015; Popov \& Kröner, 2001), the above approach is a simplification to provide a tractable solution. One additional effect is the impact of elastic anisotropy on dislocation energy, which is on the order of $10 \%$ for common geological minerals (Heinisch et al., 1975) and therefore will often be of secondary importance in situations where the lattice curvature alone dictates orders of magnitude difference in the densities of different dislocation types. Another simplification is that the energies are based on those of single dislocations rather than considering the net effects of elastically interacting groups of dislocations. The energy of groups of dislocations is a difficult problem because it depends on the precise arrangement of the dislocations but is the subject of ongoing work (Zheng et al., 2019).

The sensitivity of GND density estimates depends on three main factors, specifically, the precision $(\theta)$ in lattice rotation measurements, the mapping step size $(d)$, and the lattice orientation in the specimen reference frame. The first two factors control the precision of calculated orientation gradients and therefore the GND densities estimated from them. The precision of rotation measurements is controlled by a complex interplay of factors, including pattern size and quality along with data processing options, such as the size and number of ROIs (Britton \& Wilkinson, 2011; Wilkinson et al., 2006a). As noted above, with optimal data acquisition and processing, precision on the order of $10^{-4}$ can be achieved in practice (Britton \& Wilkinson, 2011; Wilkinson et al., 2006a). Subtle (monotonic) orientation gradients are easier to detect if the measurement points are further apart so that the orientation difference is greater. Therefore, large step sizes improve 
a)

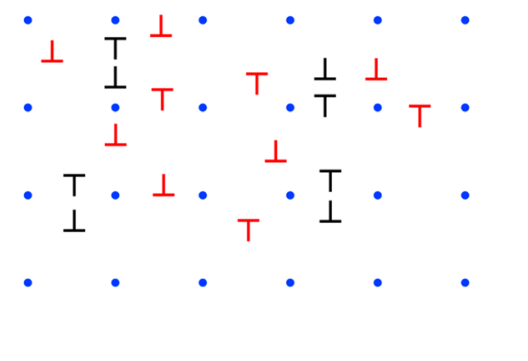

b)

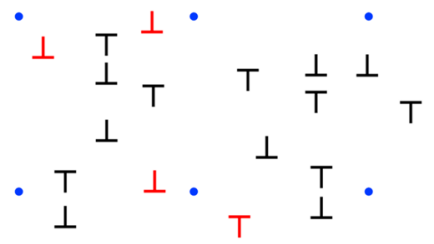

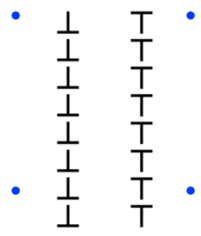

Figure 3. Schematic illustration of the effect of mapping step size on whether dislocations are evident as geometrically necessary dislocations (GNDs) or are statistically stored dislocations (SSDs). The two arrays of dislocations are identical in Figures 3a and 3b, with the only difference being the grid spacing of orientation measurements indicated as blue points. In Figure 3a most dislocations appear as GNDs. In Figure 3b the same dislocation arrangement is analyzed with a step size double that in Figure 3a, causing most dislocations to act instead as SSDs. Formally, we do not know which individual dislocation is a GND, but we do know that their net contribution must sum to the total shown in this schematic.

precision in measured orientation gradients, albeit at the expense of spatial resolution. A simple estimate of the minimum resolvable density $\left(\rho_{\min }\right)$ of GNDs with a Burgers vector of magnitude $b$ can be made by

$$
\rho_{\min }=\frac{\theta}{b d} .
$$

The minimum resolvable GND density is referred to as the noise floor because GND densities below this level are obscured by those calculated from noise in the rotation measurements (Jiang et al., 2013a; Wallis et al., 2016). The effect of lattice orientation on the noise floor in GND density estimates is more complex and has been addressed in detail by Wheeler et al. (2009) and Wallis et al. (2016). The key point is that, if the Burgers vector of an edge dislocation is orientated normal to the specimen surface, then that dislocation produces no orientation gradients detectable in the plane of observation. This effect has two main consequences. First, such dislocations cannot be detected by (mis)orientation data collected from that surface, providing one reason why GND density estimates provide a lower bound on the total dislocation density. Second, as these unfavorably oriented dislocations each produce little apparent lattice curvature, very high densities of them are required to fit noise in the measured orientation gradients. This effect results in grains with certain orientations having very high noise floors for densities of one or more types of GND.

A second reason that GND density estimates generally provide a lower bound on the total dislocation density is that some dislocation arrangements generate no net orientation gradient between measurement points. These dislocations are termed statistically stored dislocations (SSDs, Arsenlis \& Parks, 1999). A simple example is the presence of a dislocation dipole between EBSD measurement points. The opposite senses of lattice curvature of the two dislocations cause their lattice rotations to cancel over distances on the order of the spacing between the dislocations. The same effects can occur with more complex dislocation arrangements, including subgrain boundaries. If two subgrain boundaries generate lattice curvatures of equal magnitude but opposite sense between measurement points then their rotations cancel and are not detected in the orientation data. In such a data set, the dislocations that are not detected are SSDs. These effects are illustrated schematically in Figure 3, though it should be noted that it is better practice to separate the dislocation density into GND and SSD contributions rather than assigning individual defects. Clearly, the fractions of the dislocation population that appear as GNDs or SSDs are not fixed but depend on the mapping step size, dislocation arrangement, and positions of the measurement points relative to the dislocations (Jiang et al., 2013a; Ruggles, Rampton, et al., 2016; Wallis et al., 2016). In general, as step size is increased, a greater fraction of the dislocation population will become SSDs since the lattice curvature that they generate is more likely to be canceled by dislocations of the opposite sign. 


\section{Practical Implementation}

The surface preparation of specimens for HR-EBSD must be of the highest quality. With strain sensitivity on the order of $10^{-4}$, the technique is easily capable of detecting damage remaining in the specimen surface from cutting and grinding. As shifts in ROIs are measured with subpixel accuracy, any scratches visible in conventional EBSD maps based on diffraction pattern quality (e.g., band contrast, band slope, and image quality) will also be evident in the resulting HR-EBSD maps. Even very fine scratches evident in forescattered electron images during map setup are likely to be evident in the final HR-EBSD results. We typically prepare geological minerals using a standard polishing routine of progressively finer diamond suspensions, finishing with either $0.05-\mu \mathrm{m}$ diamond or colloidal silica (Lloyd, 1987), on thin, hard polishing cloths to minimize topography. However, for HR-EBSD we take the extra precaution of cleaning polishing cloths, samples, and sample holders in an ultrasonic water bath between each polishing step.

As HR-EBSD analysis is largely a postprocessing technique, most aspects of data acquisition are shared with conventional EBSD (Wilkinson \& Britton, 2012). Diffraction patterns are collected from a highly polished specimen surface tilted at $70^{\circ}$ to the incident beam and are probed with high-energy electrons. Backscattered electrons generate diffraction patterns on a phosphor scintillator screen, which is imaged using a camera based on either a charge-coupled device or complementary metal-oxide-semiconductor sensor. Optimal acquisition systems for HR-EBSD are capable of rapidly collecting and saving megapixel diffraction patterns with minimal optical distortion, and precise camera movement and positioning (Britton et al., 2013a; Maurice et al., 2013).

A few additional data acquisition steps are necessary to obtain all the information required for HR-EBSD processing. Shifts in the position of the diffraction pattern due to scanning of the beam across the specimen surface require that a correction is applied to the position of the pattern center (the point on the phosphor screen closest to the source of the diffraction pattern on the specimen surface, Figure 1; Wilkinson et al., 2006a). This correction is calculated from data obtained by scanning an undeformed single-crystal standard (typically a Si wafer), in which pattern shifts are guaranteed to result only from beam scanning and not distortion of the crystal lattice. To apply this correction and the pattern remapping procedure, the position of the pattern center must be known as accurately and precisely as possible. Some EBSD systems include an automated routine to determine the pattern center based on collecting patterns over a range of camera insertion distances (Maurice et al., 2011), which can be applied before acquisition of each data set to determine the pattern center specific to the precise specimen-camera geometry used for that experiment. Reference frame conventions used in all aspects of data acquisition and processing should be validated using the approach of Britton et al. (2016) or similar.

The optimal settings for acquisition of the diffraction patterns and the map itself depend on the aims of the analysis. Generally, for optimal strain and stress sensitivity, diffraction patterns should be collected with minimal binning and gain. Typically, shifts in ROIs caused by lattice rotations are much greater than those caused by elastic strains. Therefore, if lattice rotations or GND densities are the target of the analysis, then binning of pixels in the diffraction patterns can be applied to increase acquisition speed with negligible impact on the results, provided that the rotations are sufficiently large (Jiang et al., 2013a; Wallis et al., 2016). Map areas should be small enough that movement of the pattern center during beam scanning can be accurately corrected (Wilkinson et al., 2006a), which in practice typically limits map dimensions to a few hundred micrometers. Two main considerations, alongside the length scales of the microstructure, affect the choice of step size. First, the minimum orientation gradient and hence GND density that can be detected are inversely proportional to the step size (equation (13)). This consideration does not apply to the elastic strain and stress measurements as they are not based on spatial gradients. Second, smaller step sizes result in larger data storage requirements and longer times for acquisition and postprocessing. Typical data sets, a few hundred points in each map dimension with little or no binning of the diffraction patterns, generally require tens to hundreds of gigabytes of storage and a few days of processing on a desktop workstation. A powerful approach is to collect data sets at the highest practical spatial resolution and subsequently undersample the data points in postprocessing to systematically investigate the effect of increasing step size on GND density estimates (Jiang et al., 2013a; Ruggles, Rampton, et al., 2016; Wallis et al., 2016). Images of diffraction patterns must be saved at the maximum possible bit depth for HR-EBSD postprocessing (Britton et al., 2013a). 
Table 1

Summary of Data Sets

\begin{tabular}{lccccc}
\hline Data set & Figure (s) & $\begin{array}{c}\text { Sample } \\
\text { number }\end{array}$ & Associated publication & $\begin{array}{c}\text { Map size } \\
\text { (data points) }\end{array}$ & $\begin{array}{c}\text { Step size } \\
(\mu \mathrm{m})\end{array}$ \\
\hline Nanoindent in olivine & 4 and 5 & MN1 & (Kumamoto et al., 2017) & $258 \times 185$ & 0.2 \\
Olivine aggregate & 6 and 8 & PI-1523 & (Hansen et al., 2011) & $172 \times 116$ & 0.2 \\
Olivine single crystal & 7 & PI-1436 & (Wallis, Hansen, et al., 2017) & $450 \times 320$ & 1.0 \\
Chessboard subgrains in quartz & 9 & P13052 & (Wallis, Parsons, et al., 2017) & $92 \times 67$ & 8.0 \\
\hline
\end{tabular}

Cross-correlation analysis takes place offline and works on image files of the diffraction patterns exported from the acquisition software. The analysis is typically performed in software with image processing and matrix algebra capabilities, such as MATLAB $^{\circledR}$, or dedicated HR-EBSD packages, such as CrossCourt 4 (BLG Vantage). Requirements for running the analysis include knowledge of the pattern center position and its correction for beam scanning; knowledge of the elastic constants (for calculation of stresses and separation of normal strains) and possible dislocation types (for calculation of GND densities); selection of reference points; and choice of the size, number, and positions of the ROIs. Reference points are generally chosen within regions of high pattern quality and, where possible, that are likely to be under minimal elastic strain, as measured elastic strains are relative to the strain state of the reference point. In deformed geological materials the strain state at the reference point is typically unknown, and it is likely that there are few or no areas that are free from elastic strain. However, strains and stresses can be readily recalculated relative to the mean strain and stress state within each grain area, which is a more intuitive form to interpret and is independent of the choice of reference point (Jiang et al., 2013b; Mikami et al., 2015; Wallis, Hansen, et al., 2017). Similarly, GND density estimates are independent of the choice of reference point as they are calculated from the spatial gradients of the rotation fields.

\section{Example HR-EBSD Data Sets}

\subsection{Data Acquisition and Processing}

In this section, we present data sets that illustrate several of the main points and considerations for HREBSD analyses in general and geological minerals in particular. The data were acquired on an FEI Quanta 650 field emission gun SEM equipped with an Oxford Instruments AZtec EBSD system and NordlysNano EBSD detector in the Department of Earth Sciences, University of Oxford. Reference frames for data acquisition and processing were validated following the approach of Britton et al. (2016). The pattern center was determined prior to each run using an automated camera stepping routine in the acquisition software, implementing a process similar to that proposed by Maurice et al. (2011). Shifts in the pattern center due to beam scanning were calibrated on an undeformed single crystal Si standard (Wallis et al., 2016; Wilkinson et al., 2006a). All data sets were collected at the full resolution of the EBSD detector giving diffraction patterns of $1,344 \times 1,024$ pixels. All data sets were processed using 100 ROIs of $256 \times 256$ pixels and the robust iterative fitting and pattern remapping approaches of Britton and Wilkinson (2011, 2012b). Data points were filtered out if they had either a mean angular error $>0.004$ radians in the deformation gradient tensor or a normalized peak height $<0.3$ in the cross-correlation function (Britton \& Wilkinson, 2011). Details of the data sets are presented in Table 1. In the sections that follow, we highlight aspects of the results that are particularly relevant to the HR-EBSD method and direct interested readers elsewhere for detailed discussions of the samples and geological implications of the results.

\subsection{Lattice Rotations and Absolute Elastic Strain/Residual Stress Heterogeneities}

Figure 4 compares the precision in misorientation angles obtained by conventional EBSD and HR-EBSD processing of the same set of diffraction patterns. This data set was acquired from a nanoindent made with a triangular pyramidal (Berkovich) diamond tip in a single crystal of San Carlos olivine as part of the study by Kumamoto et al. (2017). This data set has the advantage that the olivine single crystal was well annealed with dislocation densities on the order of $<10^{10} \mathrm{~m}^{-2}$ prior to indentation (Kumamoto et al., 2017; Wallis et al., 2016). Therefore, regions of the map outside the zone of deformation around the indent are suitable for analyzing the noise levels of the measurement techniques. The maps of local misorientation, calculated 


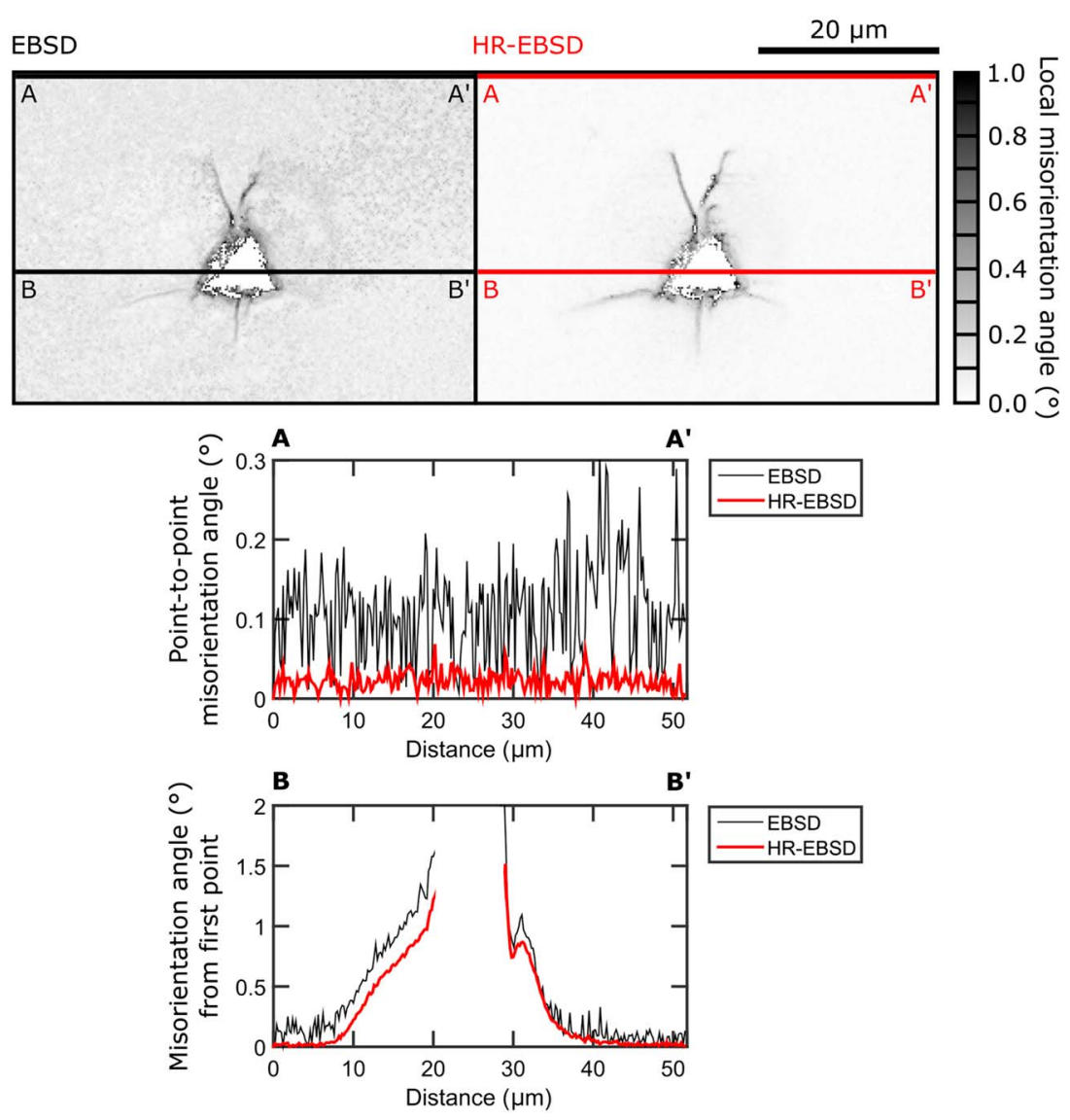

Figure 4. Misorientation data generated by conventional electron backscatter diffraction (EBSD) and high-angular resolution electron backscatter diffraction (HR-EBSD) processing of the same diffraction patterns collected across a Berkovich nanoindent in a single crystal of olivine. Local misorientation maps present the average misorientation between pixels in a $3 \times 3$ pixel kernel centered on each measurement point. Profile $\mathrm{A}-\mathrm{A}^{\prime}$ presents point-to-point misorientation angles far from the indent and therefore demonstrates the noise in the measurement methods. Profile B- ${ }^{\prime}$ presents misorientation angles relative to the orientation at point $\mathrm{B}$ across the indent and demonstrates the ability of each measurement method to reveal orientation gradients around the indent.

as the average misorientation angle within a $3 \times 3$ pixel kernel centered on each measurement point, immediately highlight the difference in noise level between the conventional EBSD and HR-EBSD data. This difference is quantified further in profile $\mathrm{A}-\mathrm{A}^{\prime}$, which presents point-to-point misorientation angles far from the indent. Two standard deviations of misorientation angles in the conventional EBSD data is $0.12^{\circ}$, which is reduced to $0.02^{\circ}$ in the HR-EBSD data. The difference that this improved precision makes to the ability to resolve subtle orientation gradients is apparent in profile $\mathrm{B}-\mathrm{B}^{\prime}$, which presents misorientation angles relative to the orientation at point $\mathrm{B}$, in a transect across the indent. The HREBSD data clearly resolve the subtle structure better than the conventional EBSD data, particularly at distances of 35-40 $\mu \mathrm{m}$ where the orientation gradient is largely obscured by noise in the conventional EBSD data.

Figure 5 presents the distributions of elastic strain and residual stress around the same nanoindent. This data set provides a useful example in which absolute values of strain and stress can be obtained by HR-EBSD because the crystal lattice at the reference point, chosen to be far from the indent, should be essentially unstrained. This assumption is supported by the uniformity of the strain fields outside the zone of influence of the indent. These far-field regions also demonstrate precision in strain and stress measurements on the order of $10^{-4}$ and a few tens of megapascals, respectively. The technique clearly resolves the strain and stress fields around the indent and linear microcracks extending from it, with magnitudes of the in-plane compressive normal stress locally exceeding $1 \mathrm{GPa}$. 


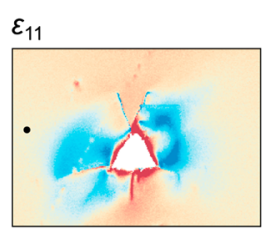

$20 \mu \mathrm{m}$

$\bar{\varepsilon}_{22}$
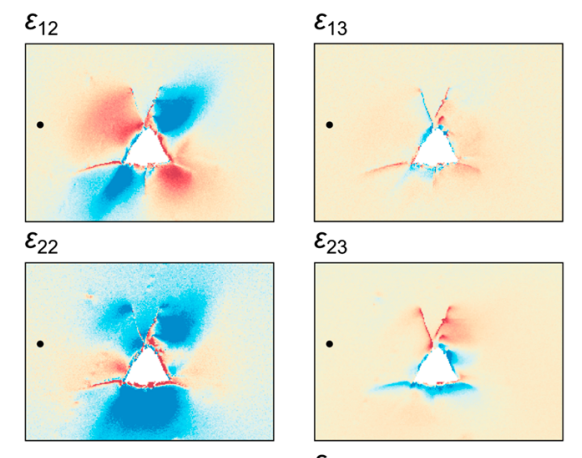

$\varepsilon_{33}$

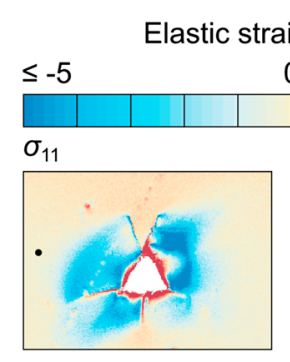

$\varepsilon_{i j}\left(x 10^{-3}\right)$

$20 \mu \mathrm{m}$

$\geq 5$

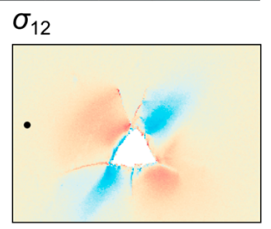

$\sigma_{22}$

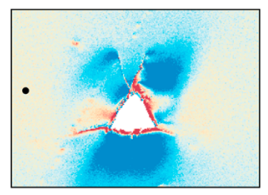

$\sigma_{23}$

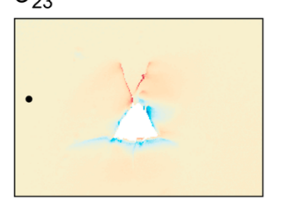

$\sigma_{33}$

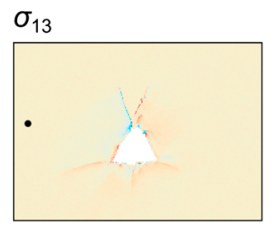

-

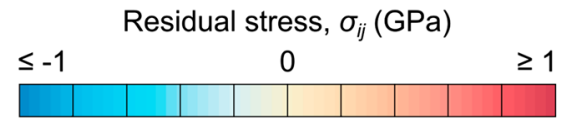

Figure 5. Maps of elastic strain $\left(\varepsilon_{i j}\right)$ and residual stress $\left(\sigma_{i j}\right)$ around the same indent as in Figure 4. All data are relative to the strain and stress states at the reference point marked in black. The $\sigma_{33}$ component of the stress tensor is constrained to be zero to enable calculation of the $\varepsilon_{33}$ component of the strain tensor (Britton \& Wilkinson, 2012b). Tensional stresses are indicated by positive values, and compressional stresses are indicated by negative values.

\subsection{Pattern Remapping and Relative Elastic Strain/Residual Stress Heterogeneities}

Figure 6 displays the effect of data processing procedures applied to data sets acquired from microstructures containing lattice rotations of several degrees, which are typical of crystalline aggregates deformed at high temperatures. This data set was obtained from an aggregate of San Carlos olivine (sample PI-1523) shortened to $17 \%$ plastic strain at temperatures in the range 1373-1523 K by Hansen et al. (2011), imparting intragranular misorientations of several degrees. In this case, it is likely that most of the material is subject to some elastic strain, as indicated by the continuously varying strain distributions. Therefore, the calculated strains, and hence stresses, are not absolute values but are relative to the unknown strain states at the reference points. Figure $6 \mathrm{a}$ presents strains measured after a single pass of cross correlation, whereas Figure $6 \mathrm{~b}$ presents strains recalculated following remapping of the test patterns into the orientations of the reference patterns and a second pass of cross correlation. Figure $6 \mathrm{c}$ presents the difference between these data sets and reveals that erroneous strains on the order of $10^{-3}$ to $10^{-2}$ were removed by the remapping procedure. This result is consistent with the work of Jiang et al. (2013b) on polycrystalline copper deformed to plastic strains of several percent. Figure $6 \mathrm{~d}$ demonstrates that the intragranular stress heterogeneities calculated from the strains after pattern remapping and the second pass of cross correlation generally still have magnitudes on the order of $1 \mathrm{GPa}$. In Figure 6e, these stresses have been recalculated by subtracting the mean value within each grain area to remove the effect of the choice of reference points (Jiang et al., 2013b; Mikami et al., 2015; Wallis, Hansen, et al., 2017). The result gives the intragranular stress heterogeneities relative to the (unknown) mean stress state within each grain area.

\subsection{Geometrically Necessary Dislocations}

5.4.1. Comparison Between GND Densities From Conventional EBSD and HR-EBSD

Figure 7 presents a comparison of GND densities in a single crystal of olivine calculated from orientation gradients derived from conventional EBSD and HR-EBSD analysis of the same diffraction patterns. The crystal was experimentally deformed at $1473 \mathrm{~K}$ with the compression direction running vertically in the maps. Wallis, Hansen, et al. (2017) discussed the HR-EBSD results in detail, whereas here we focus on their relationship to the conventional EBSD data. GND densities were estimated using the $L_{2}$ method (equation (10)) of Wallis et al. (2016). In both data sets, the most obvious set of structures are bands of elevated GND density trending top right to bottom left. Less prominent arrays of linear features trending top left to bottom right result from small rotations across microcracks (Wallis, Hansen, et al., 2017). Two key differences are evident between the results obtained from conventional EBSD and HR-EBSD. First, the noise level is higher in the conventional EBSD data, particularly on the left side of the map where the details of the GND structures are partially obscured. This contrast is an obvious outcome of the different angular resolutions of the two methods. Second, the dislocation types to which the GND densities are assigned differ between the two data sets. Densities of [100] screw, [001] screw, and (010)[001] edge dislocations are higher in the results from conventional EBSD than in the results from HR-EBSD, while the opposite is true of the density of (001)[100] edge dislocations. This difference is a consequence of the different misorientation axes determined by the two methods, with those from HR-EBSD being the more precise (Wilkinson, 2001).

5.4.2. The Effect of Crystal Orientation on Noise Floors in GND Density Estimates

Figure 8 illustrates the effect of crystal orientation on the noise floors in GND density estimates using the same data set as in Figure 6. This aggregate of olivine contains grains in a variety of orientations. Figure 8 

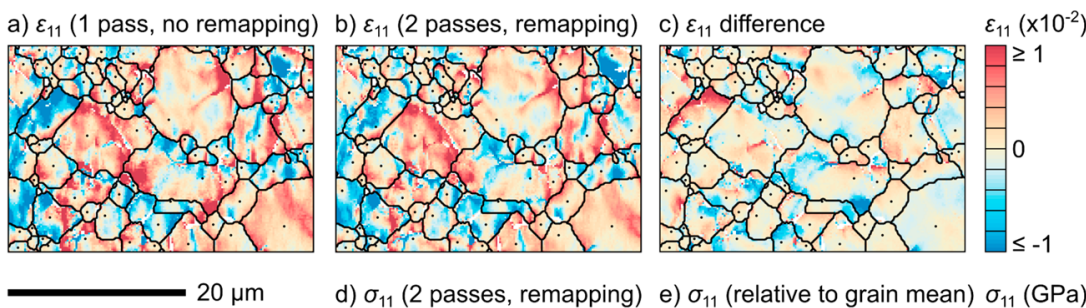

d) $\sigma_{11}$ (2 passes, remapping)

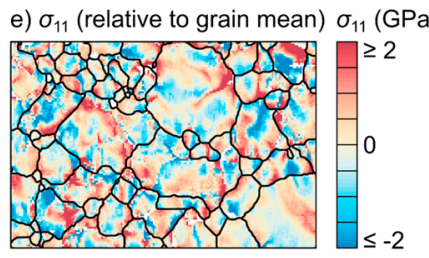

Figure 6. The effect of data processing procedures on measured elastic strains and residual stresses. The top row displays the $\varepsilon_{11}$ component of the strain tensor (a) after one pass of cross correlation, (b) after two passes of cross correlation incorporating remapping of the test patterns into the orientations of the reference patterns, and (c) the difference between Figures $6 \mathrm{a}$ and $6 \mathrm{~b}$. Black lines indicate grain boundaries with $\geq 10^{\circ}$ misorientation. Black dots indicate the reference point for each grain. The bottom row displays the $\sigma_{11}$ component of the stress tensor (d) computed after two passes of cross correlation and pattern remapping (i.e., corresponding to the strains in Figure 6b) and (e) recalculated relative to its mean value within each grain.

a color codes these orientations according to the crystal direction oriented normal to the specimen surface. Figure $8 \mathrm{~b}$ presents estimates of the noise floor for densities of (010)[100] edge dislocations in these differently oriented grains. To estimate the noise floor, we estimated the precision in orientation gradients $(\phi)$ from the angular resolution of the HR-EBSD measurements $(\theta)$ and the step size $(d)$ by

$$
\phi=\frac{\theta}{d}
$$

We estimated $\theta$ to be $3 \times 10^{-4}$ rad for this data set based on the results of Wilkinson et al. (2006a) and Wallis et al. (2016), the diffraction pattern size in Table 1, and comparison of the estimated noise floors to Figure 8c. To predict the GND density noise floors in each grain, we used the crystal orientations of each reference point along with the estimate of $\phi$, instead of measured orientation gradients, as inputs for the same $L_{2}$ minimization procedure (equation (9)) applied to the real data. Edge dislocations with [100] Burgers vectors produce little/no detectable lattice curvature when [100] is oriented (sub)normal to the specimen surface, and therefore, high densities of them are required to fit the orientation noise (section 3.2). One example of a grain in this orientation is marked with a black star in Figures 8a-8c. Grains with [100] axes at lower angles to the specimen surface generally have lower estimated noise floors for densities of (010) [100] edge dislocations. The measured densities of (010)[100] edge dislocations are presented in Figure 8c. Grains, including the marked example, with high estimated noise floors in Figure 8b generally also exhibit high GND densities in Figure 8c, obscuring any GND structures. Most other grains in this specimen exhibit distinct GND structures, including patches and bands of elevated GND density, resolvable above the noise floor.

\subsubsection{Minerals With More Than Six Dislocation Types}

In the examples above, dislocation types could be separated using the $L_{2}$ approach (section 3.2) because olivine has relatively few slip systems active at high temperature, and therefore considering six dislocation types provides a reasonable characterization and a unique solution. However, many geological minerals, particularly those with higher symmetry, have many more dislocation types that can plausibly be activated (as is typically the situation in metallurgical studies). Important examples include quartz (Linker et al., 1984; Lloyd et al., 1997), calcite (de Bresser \& Spiers, 1997), and garnet (Mainprice et al., 2004). For cubic minerals, in certain applications it may be appropriate to assume a dominant family of slip systems and solve for an optimized density of each of the associated symmetrically equivalent dislocation types, as has been carried out for cubic metals (Wilkinson \& Randman, 2010). For body centered cubic garnet, consideration of 16 dislocation types (12 edge and 4 screw) on the $\{110\}<111>$ slip system could provide a reasonable approximation (Mainprice et al., 2004). This approach was taken by Wilkinson and 


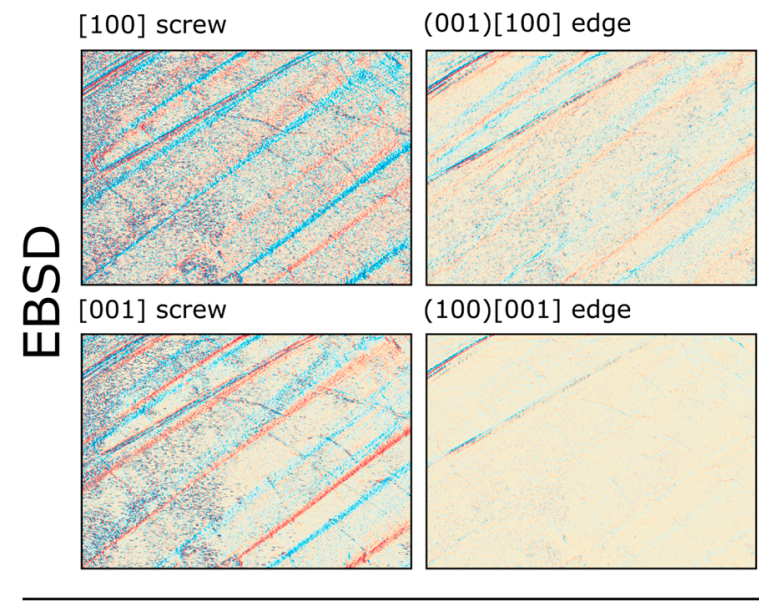

$(010)[100]$ edge

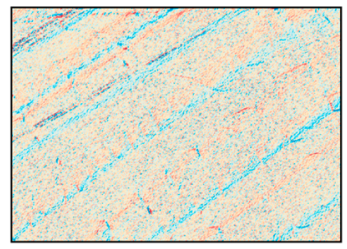

(010)[001] edge
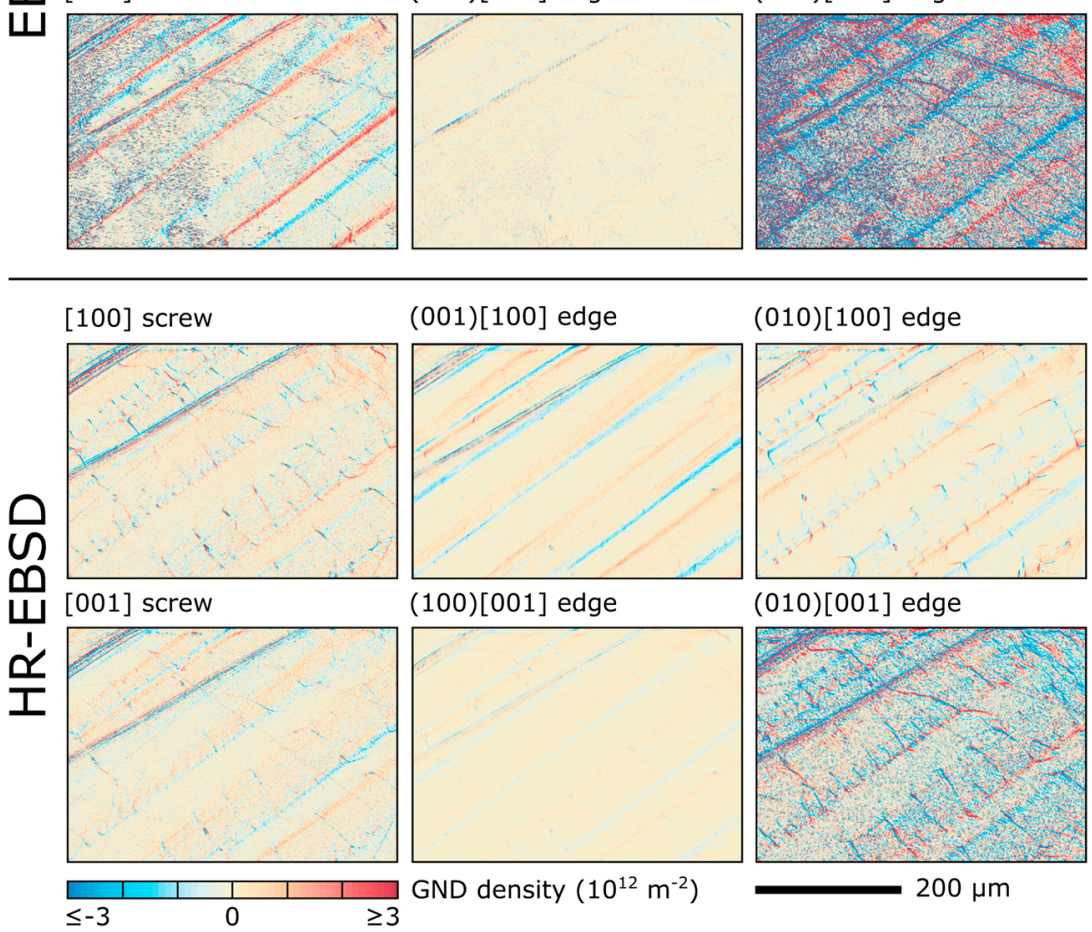

(010)[100] edge

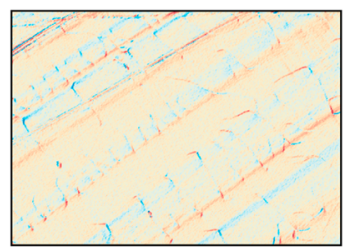

(010)[001] edge

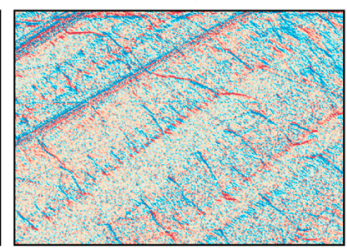

$200 \mu \mathrm{m}$

Figure 7. Densities of six types of geometrically necessary dislocation in a single crystal of olivine calculated from lattice orientation gradients obtained by conventional EBSD (top) and HR-EBSD processing of the same diffraction patterns (bottom). Positive and negative GND densities indicate dislocations with opposite senses of Burgers vector (i.e., generating opposite senses of lattice curvature). HR-EBSD = high-angular resolution electron backscatter diffraction; EBSD = electron backscatter diffraction; GND = geometrically necessary dislocation.

a) IPF, $Z$ direction

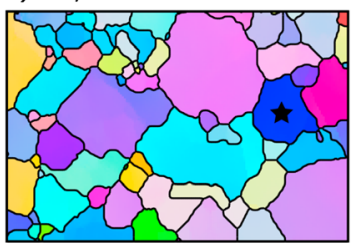

[100]

$[001]$

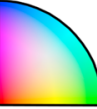

b) Estimated noise floor
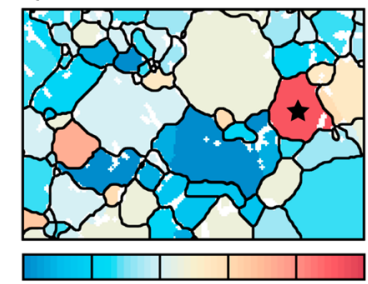$$
10^{12}
$$

$10^{12}$

[010]
GND density $\left(\mathrm{m}^{-2}\right)$,

(010)[100] edge dislocations c) Mapped GND density

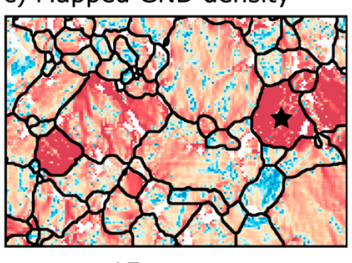

$15 \mu \mathrm{m}$

Figure 8. The effect of crystal orientation on the noise floor of geometrically necessary dislocation (GND) density estimates. (a) Map color coded for crystal orientation according to the inverse pole figure (IPF) for the $Z$ direction of the specimen (i.e., the normal to the specimen surface). (b) Estimated noise floor for densities of (010)[100] edge GNDs. (c) Mapped densities of (010)[100] edge GNDs. The black star marks a grain with [100] oriented normal to the specimen surface. Black lines indicate grain boundaries with misorientation angles $\geq 10^{\circ}$. 


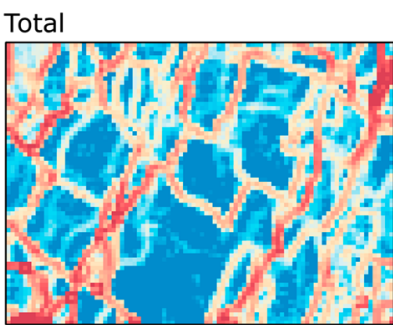

(c) $<a>$ edge

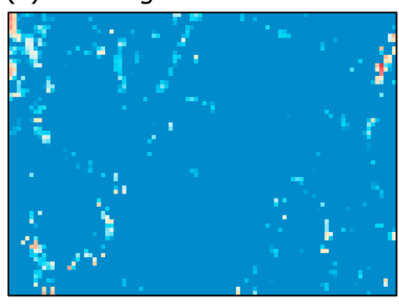

$\{m\}<a>$ edge

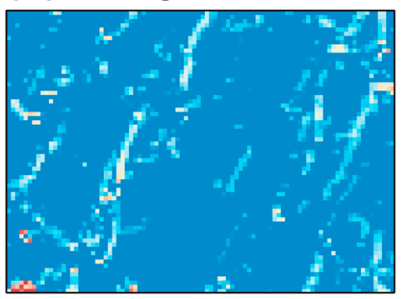

$<a>$ screw

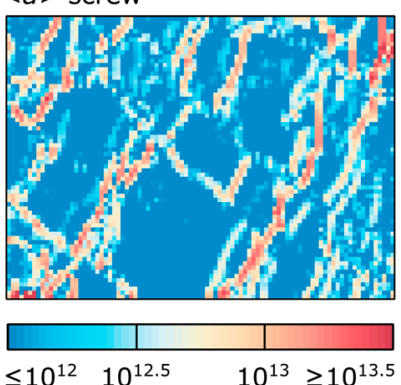

$\leq 10^{12} \quad 10^{12.5} \quad 10^{13} \geq 10^{13.5}$
$N$
$O$
$O$
$ㄷ$

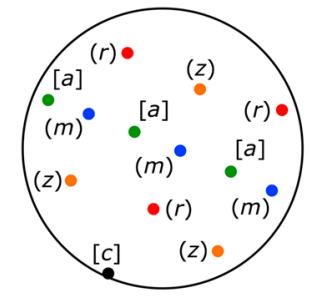

$\{r / z\}<a>$ edge

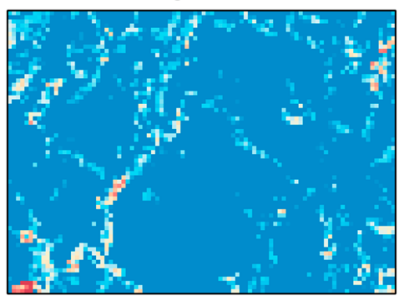

$\{m\}[c]$ edge

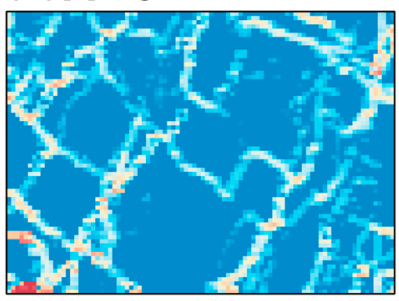

[c] screw

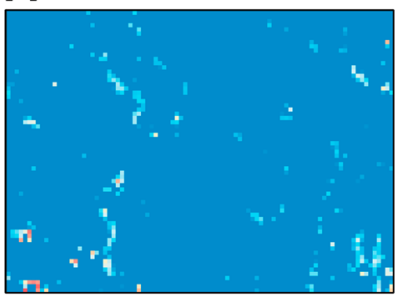

GND density $\left(\mathrm{m}^{-2}\right)$

Figure 9. Geometrically necessary dislocation (GND) densities in chessboard subgrain boundaries in quartz from the Greater Himalayan Sequence, Nepal. The pole figure indicates the crystal orientation. From Wallis, Parsons, et al. (2017).

Randman (2010) in their analysis of the GND content of body centered cubic $\mathrm{Fe}$, in which they selected a solution for the densities of each dislocation type by employing the $L_{1}$ scheme to minimize the total line energy (equation (11)). In geological examples of such analyses, the sum of the densities of the symmetrically equivalent dislocation types will often be the result of interest, and their subdivision may be less important.

The situation is more complex for minerals, such as trigonal quartz and calcite, with more than six dislocation types that are spread across multiple families of slip systems. In such cases, typically the family of slip systems cannot be assumed a priori and instead is the information of interest. Moreover, the abundance of dislocation types generates considerable redundancy in solving equation (8), and again the $L_{1}$ scheme must be employed to minimize some other variable, such as the total line energy, but now the specific dislocation types favored by the minimization are of importance. This approach has been employed for hexagonal close packed metals, such as Ti (Britton, Birosca, et al., 2010; Britton, Maurice, et al., 2010; Britton \& Wilkinson, 2012a). Wallis, Parsons, et al. (2017) adopted this method in analyses of chessboard subgrain boundaries in quartz, in which they considered the six families of dislocation types with either $\langle a>$ or $[c]$ Burgers vectors presented in Figure 9. Transmission electron microscope and visible light microscope observations of chessboard subgrain boundaries indicate that they are composed primarily of $\{m\}[c]$ and $(c)<a>$ edge dislocations (Blumenfeld et al., 1986; Kruhl, 1996; Mainprice et al., 1986; Okudaira et al., 1995). In the HR-EBSD results in Figure 9 and the other samples analyzed by Wallis, Parsons, et al. (2017), $\{m\}[c]$ edge dislocations are abundant, but $(c)\langle a\rangle$ edge dislocations are largely absent. Instead, $<a>$ screw dislocations are apparent in high densities, particularly in boundaries with traces parallel to [0001], which would otherwise be expected to be composed of $(c)<a>$ edge dislocations. Wallis, Parsons, et al. (2017) attributed the difference between the HR-EBSD results and previous results to the lower energy of screw dislocations relative to edge dislocations, which results in them being favored in the energy minimization scheme. Wilkinson and Randman (2010) also noted that the $L_{1}$ energy minimization scheme returned greater densities of screw dislocations than edge dislocations in their analysis of Fe. Despite these complications, Wallis, Parsons, et al. (2017) highlighted that it is possible to unambiguously discriminate dislocations with $<a>$ Burgers vectors from those with $[c]$ Burgers vectors using this approach.

\section{Strengths of HR-EBSD for Analyzing Intracrystalline Lattice Distortion}

HR-EBSD has several obvious advantages over conventional, Hough transform-based, EBSD for analysis of intragranular lattice distortions. These advantages stem from the improved precision in misorientation angles and axes, along with the ability to map heterogeneities in elastic strain and residual stress (Britton \& Wilkinson, 2012b; Wilkinson, 1996, 2001; Wilkinson et al., 2006a). These capabilities have been widely exploited in the materials sciences (Britton, Birosca, et al., 2010; Britton, Maurice, et al., 2010; Britton \& Wilkinson, 2012a; Jiang et al., 2013b, 2013c; Jiang, Britton, et al., 2015; Jiang et al., 2016; Littlewood et al., 2011; Maurice et al., 2013; Villanova et al., 2012; Vilalta-Clemente et al., 2017; Wilkinson \& Britton, 2012). However, geological materials present a diverse new array of crystal structures, microstructures, deformation mechanisms, and conditions of formation and deformation, which can be investigated using HR-EBSD. Therefore, in this section we discuss in a general sense the benefits that HR-EBSD brings to analysis of geological minerals in particular. 
Many deformation microstructures relevant to geological interpretations involve very small misorientation angles. Some of these microstructures, including deformation lamellae (Trepmann \& Stöckhert, 2003), slip bands (de Bresser, 2002), and undulose extinction (Halfpenny et al., 2006), are the subtle expressions of limited dislocation activity at low homologous temperatures. Other common microstructures form at high homologous temperatures, at which differential stresses and hence dislocation densities are often low, and include incipient subgrain boundaries (Lloyd et al., 1997; Wheeler et al., 2009; Wallis, Parsons, et al., 2017) and low densities of free dislocations in subgrain interiors (de Bresser, 1996; Qi et al., 2018). Analyses of these microstructures clearly benefit from the precision in misorientation angles offered by HR-EBSD. This effect is demonstrated in Figures 4 and 7, where the subtleties of microstructures formed at low and high temperatures, respectively, are revealed in new detail by HR-EBSD processing of the diffraction data. Moreover, the improved misorientation axes provided by HR-EBSD (Wilkinson, 2001) make it possible to reliably investigate the components of lattice curvature and hence types of dislocations contributing to these microstructures. The full benefits of improved misorientation axes are realized in applications to geological minerals due to the diversity of dislocation types and associated slip systems that they exhibit (e.g., Figures 7 and 9). Deformation of high-symmetry crystal structures, such as the cubic metals, is generally accommodated primarily by one family of symmetrically equivalent dislocation types (Jiang et al., 2013c; Wilkinson \& Randman, 2010), reducing the need to discriminate between them. The generally lower symmetries of geological materials typically require that more than one family of dislocation types are required to be active to accommodate an arbitrary deformation (Detrez et al., 2015; Morales et al., 2014), and their associated slip systems typically have different strengths (Linker et al., 1984; Bai et al., 1991; de Bresser \& Spiers, 1997). Therefore, determining the dislocation types and slip systems that were active is often one of the principal goals of geological studies. These analyses require precise misorientation axes to correctly populate the Nye tensor, $\mathbf{a}$, in equation (6) and $\lambda$ in equation (8). This benefit of HR-EBSD processing is highlighted in Figure 7, in which the dominant types of dislocation forming the substructure differ between the conventional EBSD and HR-EBSD data. Complementary approaches to analyzing the dislocation content based on the Nye tensor, such as the weighted Burgers vector of Wheeler et al. (2009), could benefit similarly from taking HR-EBSD misorientation data as inputs.

Alongside generating lattice misorientations, the diverse and complex histories of many geological materials involve many potential sources of heterogeneous residual stress and elastic strain (Friedman, 1972; Holzhausen \& Johnson, 1979). Structural defects in rocks are one source of residual stress heterogeneity and include dislocations (Anderson et al., 2017) and microcracks (Sun \& Jin, 2012). Another diverse group of sources encompasses residual stresses arising from interactions among grains with anisotropic properties. An aggregate of grains that are elastically and plastically anisotropic can have stresses locked in during deformation as the shape changes of the grains prevent full relaxation upon removal of the macroscopic applied load (Friedman, 1972). Rocks can accrue additional residual stress heterogeneity during decompression and cooling due to anisotropic elastic properties and thermal expansivities, respectively (Holzhausen \& Johnson, 1979; Rosenfeld \& Chase, 1961). Similarly, phase changes potentially introduce stress heterogeneity as a result of changes in volume, which drive additional processes, such as transformation plasticity (Poirier, 1982). HR-EBSD can also map changes in the relative lengths of unit cell axes resulting from solid solutions (Schäffer et al., 2014; Speller et al., 2014). These compositional variations manifest as pseudostrain heterogeneity due to variations in interplanar angles.

Interpretation of the geological processes recorded in residual stress fields has been hindered by the challenge of measuring elastic strains and residual stresses at the grain scale. Techniques that are currently employed include Raman spectroscopy (Kohn, 2014) and X-ray Laue microdiffraction (Boullier et al., 2017; Chen et al., 2015, 2016). Another technique that could potentially be employed is convergent beam electron diffraction in the transmission electron microscope (Champness, 1987). HR-EBSD has advantages over each of these techniques that make it a particularly appealing option. While Raman spectroscopy can be applied to unrelaxed grains confined within a specimen volume, it provides only a scalar measure of confining pressure, rather than the full stress tensor. Absolute values of the full stress tensor can be obtained from X-ray Laue microdiffraction, but such measurements require access to a synchrotron X-ray source, making routine analysis of large sample sets challenging. In contrast, HR-EBSD provides a method to obtain broadly comparable data using a standard SEM. Moreover, HR-EBSD offers improved spatial resolution (potentially $<100 \mathrm{~nm}$ ) relative to Raman spectroscopy and X-ray diffraction (Dingley et al., 2010). Transmission electron 
microscopy provides even greater spatial resolution but at the expense of areal extent and at the risk of greater modification of the stress state during specimen preparation (Dingley et al., 2010). HR-EBSD provides a combination of precision, spatial resolution, and areal coverage that are well suited to the microstructures of deformed rocks with characteristic length scales in the range $10^{-7}$ to $10^{-4} \mathrm{~m}$.

As with all microstructural data, the interpretation of HR-EBSD results is not trivial. Mapped GND density and stress fields record the net effect of potentially complex deformation histories that may include one or more deformation events of interest as well as additional processes, such as generation of stresses during decompression and cooling. These challenges apply to samples deformed in both nature and experiments. However, these challenges are not specific to HR-EBSD data but apply also to microstructural data acquired by any technique. Rather, the improved level of microstructural detail provided by the precision of HR-EBSD can only aid in the interpretation of processes that controlled microstructural evolution during the history of the rock. For example, the ability to resolve dislocation structures associated with subtle misorientations, to provide new constraints on the dislocation types that contribute to them, and assess their association with stress heterogeneities helps to constrain the processes of microstructural evolution in both experimental (e.g., Wallis, Hansen, et al., 2017) and natural (e.g., Wallis et al., 2017b) samples. Ongoing and future work will focus on developing strategies for deciphering the contributions of different causes to heterogeneous residual stress fields.

\section{Current Limitations of HR-EBSD and Areas for Further Development}

Several caveats should be borne in mind when designing HR-EBSD experiments and interpreting HR-EBSD data. Some of these considerations stem from the data processing procedures and are therefore specific to HR-EBSD. However, several key points are inherent to (mis)orientation and stress data sets more generally, regardless of the technique used to acquire the data.

The characteristics of a microstructure can place constraints on the information that can be revealed by HREBSD analysis. A limitation of current data processing procedures is that crystal orientations at a given pixel must be within $11^{\circ}$ of the crystal orientation at the reference point (Britton \& Wilkinson, 2011, 2012b). Beyond this range, the patterns to be compared are too dissimilar and distorted for reliable cross-correlation analysis. This constraint limits the maximum intragranular orientation range that can be analyzed with one reference point to $22^{\circ}$, assuming that a reference point with the optimal orientation can be found in advance. Grains within rocks deformed to large plastic strains at high homologous temperatures commonly develop arrays of subgrains that can result in intragranular orientation ranges $>22^{\circ}$ (e.g., Cross et al. (2017)). In such cases, more than one reference point must be chosen (e.g., one reference point per subgrain) and stress states will generally not be directly comparable between the areas associated with each reference point. It is possible that this limitation could be overcome by developing a routine for cross correlating between two or more reference points chosen to be within $11^{\circ}$ of one another. For example, if the stress states of two points with orientations differing by $10^{\circ}$ were cross referenced by the cross-correlation procedure, and both those points were subsequently used as reference points, then the orientation range could be extended to $32^{\circ}$. However, this procedure has not yet been applied in practice.

The orientation in which a specimen is sectioned can limit or optimize the information recovered. Some sections through a crystal are better than others for revealing the lattice curvatures induced by possible dislocation types (Wallis et al., 2016; Wheeler et al., 2009). Poorly oriented sections may not reveal lattice curvature generated by an important dislocation type and may result in high noise floors in estimates of GND density, as illustrated in Figure 8. Importantly, this caveat applies not only to HR-EBSD data but also to conventional EBSD and all other orientation data collected on two-dimensional sections. Fortunately, often specimens can be deliberately sectioned in an optimal orientation for revealing the dislocation content based on a priori knowledge of the crystal orientation or interpretation of the likely crystallographic preferred orientation. This approach has been applied to single crystals (Wallis et al., 2016; Wallis, Hansen, et al., 2017) and aggregates (Qi et al., 2018) of olivine, which can be sectioned such that the [100] and [001] Burgers vectors generally lie at low angles to the plane of the section. Similar considerations apply to analysis of stress heterogeneity as the stress state is modified by sectioning of the sample. In particular, the normal stress on the surface is relaxed, while the other stress components are modified to a lesser extent due to the Poisson effect and changes in the tractions on the surface (Kartal et al., 2015; Pagliaro et al., 2011). However, if a 
specific component of the stress state is of particular interest (e.g., the shear stresses acting on a slip system), then a section orientation can be chosen that minimizes the extent to which that component is modified during sectioning. A complimentary approach, which has not yet been exploited in HR-EBSD analysis of geological materials, would be to analyze mutually perpendicular sections. The combined data set would provide, at least in a statistical sense, a more complete characterization of the orientation gradients (i.e., the GND content), along with differential relaxation of each stress component in each section. To date, fully three-dimensional HR-EBSD, based on serial sectioning, has not been achieved due to the difficulty of aligning the sections with sufficient precision and correcting changes in pattern center position.

Several approaches to interpreting the types and densities of GNDs from orientation gradients are available, and each is associated with advantages and disadvantages. The method most widely employed in analysis of conventional EBSD data is to plot misorientation axes on an inverse pole figure and to interpret the dislocation types most likely to have generated the misorientations (Bestmann \& Prior, 2003; Lloyd et al., 1997; Prior et al., 2002). While this approach is simple and intuitive, it has the drawbacks of being qualitative, being difficult to decipher the combined effects of multiple dislocation types, and relying on (often implicit) assumptions about the possible dislocation types. A method that has the benefits of being quantitative and not relying on assumptions about possible dislocation types is the weighted Burgers vector approach of Wheeler et al. (2009), which employs the fully constrained $a_{i 3}$ components of the Nye tensor. However, this analysis does not exploit the additional constraints on the dislocation content that can be gleaned from other components of the Nye tensor if gradients in elastic strain are assumed to be small (as they are in all analyses based on conventional EBSD, in which elastic strains are not measured). In contrast, the approach outlined in section 3.2 exploits all of the available orientation gradients, potentially providing a more complete description of the dislocation content, but requires assumptions about the possible dislocation types in order to find their best fit densities. Furthermore, cases in which more than six dislocation types must be considered require an additional assumption, such as dislocations occupying a minimum-energy configuration, to choose a single solution to equation (8). As the presence of dislocations implies that their energy is not at a minimum, minimizing energy in equation (11) may not always give an appropriate solution, as suggested by the analysis of quartz in section 5.4.3 and Wallis, Parsons, et al. (2017). Nonetheless, the method outlined in section 3.2 does provide a fully quantitative approach that exploits all available components of the orientation gradients, and in which all assumptions are made fully explicit. We emphasize that each of the above approaches to GND density analysis could be applied to orientation data collected by any method, and therefore, their strengths and limitations are not related to the method by which the orientation data were acquired (e.g., conventional EBSD, HR-EBSD, and X-ray diffraction). Instead, all analyses of intragranular misorientations can benefit from the precise rotation data offered by the cross-correlation approach of HR-EBSD.

A notable limitation of elastic strain and residual stress data from HR-EBSD is that absolute measurements can only be obtained if unstrained material is present to provide a reference point. This condition is met in particular situations, such as the nanoindent in section 5.2 (Figure 5). However, in most rocks no material can be assumed to be free from elastic strain. Therefore, generally HR-EBSD provides maps of relative heterogeneities in elastic strain and residual stress, as in section 5.3. Nonetheless, although the strain state of reference points is generally unknown, the data can be normalized relative to the mean value of each strain/stress component in each grain (Figure 6), providing values that are potentially more intuitive to interpret (Mikami et al., 2015). An additional complication is the diversity of potential sources of residual stress in geological materials (section 6), which makes it challenging to decipher the contributions from particular processes. Fortunately, many geological applications can still benefit from comparisons of relative stress states; for instance, whether stresses are more heterogeneous in different minerals or between different rocks. Such comparisons may also be exploited to constrain causes of stress heterogeneity, for instance, comparing a rock that has undergone deformation and exhumation to one that has only undergone equivalent exhumation.

Recently, some new approaches to HR-EBSD have been proposed, which attempt to simplify the procedure and improve accuracy by using more advanced digital image correlation (DIC) methodologies. Ruggles et al. (2018) suggested the use of inverse compositional Gauss-Newton DIC to track the changes in shape of ROIs along with shifts in their positions. Similarly, Vermeij and Hoefnagels (2018) have developed a method that uses finite-strain integrated DIC to correlate the full diffraction patterns in one step, circumventing the use 
of ROIs and pattern remapping. Recently, Vermeij et al. (2019) extended this approach to suggest that simultaneous correlation of all overlapping areas in multiple diffraction patterns can, in theory, be exploited to optimize crystal orientation, stress state, and pattern center coordinates, providing measurements of absolute stress state extending across multiple grains. So far, these approaches appear promising in tests on simulated diffraction patterns but have not been applied to, or rigorously tested on, experimental diffraction patterns.

\section{Promising Targets for HR-EBSD Analysis}

The new capabilities offered by HR-EBSD make it easy to envision applications in many areas of rock deformation and petrology. The precise characterization of dislocation content and associated stress fields is ideally suited to applications in high-temperature rock deformation, which has been the focus of most initial investigations (Boneh et al., 2017; Kumamoto et al., 2017; Qi et al., 2018; Wallis et al., 2016, Wallis, Hansen, et al., 2017; Wallis, Parsons, et al., 2017). Specimens deformed in laboratory experiments can be analyzed using HR-EBSD to inform models of deformation processes and potentially to identify microstructures diagnostic of particular rheological behaviors. Natural specimens can be subjected to similar analysis to assess the applicability of laboratory-based models. Similar applications will likely be found in investigations of dynamic and static recrystallization (e.g., Boneh et al., 2017) and palaeopiezometry. A key advantage of HR-EBSD in these efforts is the capability to provide quantitative data on both GND densities and residual stresses over length scales in the range $10^{-1}$ to $10^{2} \mu \mathrm{m}$. This length scale is sufficient to span multiple grains in most deformed rocks and is therefore ideal for bridging the scales between transmission electron microscopy, which can image individual dislocations and map strain at higher spatial resolutions, and more representative rock volumes.

HR-EBSD will likely also be useful in studies of deformation at low temperatures. Stress concentrations associated with compaction or fracturing are ideal targets. HR-EBSD has been applied in studies of crack nucleation in nickel-based superalloys (Jiang, Yang, et al., 2015; Zhang et al., 2015), and stress concentrations around the tips of microcracks have been observed in olivine single crystals (Wallis, Hansen, et al., 2017). Deformation at shallow depths and low temperatures should limit the stress heterogeneities resulting from exhumation and cooling, potentially aiding recognition of stress heterogeneities recording prior deformation.

HR-EBSD could also prove useful in petrological studies by revealing intragranular deformation associated with changes in phase and pressure-temperature conditions. Promising targets include deformation around solid and fluid inclusions in mineral grains (Angel et al., 2014; Avadanii et al., 2017) and due to crystal growth or phase transformations (Gardner et al., 2017; van Noort et al., 2017).

\section{Conclusions}

HR-EBSD is a promising technique developed in the materials sciences that has recently been exploited in initial applications to geological materials. The capabilities of the technique make it well suited to analysis of intragranular lattice distortions of deformed minerals. Its key strength is the ability to map lattice rotations and elastic strains with precision on the order of $10^{-4}$ and submicron spatial resolution in a SEM. These data provide the bases for estimates of GND density and calculation of residual stress heterogeneity. Caveats include the complex factors that influence GND density estimates, the effect of sectioning on stress state, and that maps of stress heterogeneity, rather than absolute stress state, are obtained from most materials. Nonetheless, the depth of information obtained from HR-EBSD promises new insights and advances in many areas of rock deformation and petrology in both laboratory and natural contexts.

\section{References}

Adams, B. L., Wright, S. I., \& Kunze, K. (1993). Orientation imaging: The emergence of a new microscopy. Metallurgical Transactions A, 24(4), 819-831. https://doi.org/10.1007/bf02656503

Anderson, P. M., Hirth, J. P., \& Lothe, J. (2017). Theory of Dislocations. Cambridge: Cambridge University Press.

Angel, R. J., Mazzucchelli, M. L., Alvaro, M., Nimis, P., \& Nestola, F. (2014). Geobarometry from host-inclusion systems: The role of elastic relaxation. The American Mineralogist, 99(10), 2146-2149. https://doi.org/10.2138/am-2014-5047

Arsenlis, A., \& Parks, D. M. (1999). Crystallographic aspects of geometrically-necessary and statistically-stored dislocation density. Acta Materialia, 47(5), 1597-1611. https://doi.org/10.1016/s1359-6454(99)00020-8 
Avadanii, D., Hansen, L., Wallis, D., \& Waters, D. (2017). A new barometer from stress fields around inclusions. 19th EGU General Assembly. 1073.

Bai, Q., Mackwell, S. J., \& Kohlstedt, D. L. (1991). High-temperature creep of olivine single crystals 1. Mechanical results for buffered samples. Journal of Geophysical Research, 96, 2441. https://doi.org/10.1029/90jb01723

Bestmann, M., \& Prior, D. J. (2003). Intragranular dynamic recrystallization in naturally deformed calcite marble: Diffusion accommodated grain boundary sliding as a result of subgrain rotation recrystallization. Journal of Structural Geology, 25(10), 1597-1613. https://doi.org/ 10.1016/s0191-8141(03)00006-3

Blumenfeld, P., Mainprice, D., \& Bouchez, J. L. (1986). C-slip in quartz from subsolidus deformed granite. Tectonophysics, 127, 97-115. https://doi.org/10.1016/0040-1951(86)90081-8

Boneh, Y., Wallis, D., Hansen, L. N., Krawczynski, M. J., \& Skemer, P. (2017). Oriented grain growth and modification of "frozen anisotropy" in the lithospheric mantle. Earth and Planetary Science Letters, 474, 368-374. https://doi.org/10.1016/j.epsl.2017.06.050

Boullier, A.-M., Robach, O., Ildefonse, B., Barou, F., Mainprice, D., Ohtani, T., \& Fujimoto, K. (2017). High stresses stored in fault zones: example of the Nojima fault (Japan). Solid Earth Discussions, 1-41. https://doi.org/10.5194/se-2017-130

de Bresser, J. H. P. (1996). Steady state dislocation densities in experimentally deformed calcite materials: Single crystals versus polycrystals. Journal of Geophysical Research, 101(B10), 22,189-22,201. https://doi.org/10.1029/96jb01759

de Bresser, J. H. P. (2002). On the mechanism of dislocation creep of calcite at high temperature: Inferences from experimentally measured pressure sensitivity and strain rate sensitivity of flow stress. Journal of Geophysical Research, 107(B12), 2337. https://doi.org/10.1029/ 2002jb001812

de Bresser, J. H. P., \& Spiers, C. J. (1997). Strength characteristics of the r, f, and c slip systems in calcite. Tectonophysics, 272(1), 1-23. https://doi.org/10.1016/s0040-1951(96)00273-9

Britton, T. B., Birosca, S., Preuss, M., \& Wilkinson, A. J. (2010). Electron backscatter diffraction study of dislocation content of a macrozone in hot-rolled Ti-6Al-4V alloy. Scripta Materialia, 62(9), 639-642. https://doi.org/10.1016/j.scriptamat.2010.01.010

Britton, T. B., Jiang, J., Clough, R., Tarleton, E., Kirkland, A. I., \& Wilkinson, A. J. (2013a). Assessing the precision of strain measurements using electron backscatter diffraction-Part 1: Detector assessment. Ultramicroscopy, 135, 126-135. https://doi.org/10.1016/j. ultramic.2013.08.005

Britton, T. B., Jiang, J., Clough, R., Tarleton, E., Kirkland, A. I., \& Wilkinson, A. J. (2013b). Assessing the precision of strain measurements using electron backscatter diffraction-Part 2: Experimental demonstration. Ultramicroscopy, 135, 136-141. https://doi.org/10.1016/j. ultramic.2013.08.006

Britton, T. B., Jiang, J., Guo, Y., Vilalta-Clemente, A., Wallis, D., Hansen, L. N., et al. (2016). Tutorial: Crystal orientations and EBSD—Or which way is up? Materials Characterization, 117, 113-126. https://doi.org/10.1016/j.matchar.2016.04.008

Britton, T. B., Maurice, C., Fortunier, R., Driver, J. H., Day, A. P., Meaden, G., et al. (2010). Factors affecting the accuracy of high resolution electron backscatter diffraction when using simulated patterns. Ultramicroscopy, 110(12), 1443-1453. https://doi.org/10.1016/j. ultramic.2010.08.001

Britton, T. B., \& Wilkinson, A. J. (2011). Measurement of residual elastic strain and lattice rotations with high resolution electron backscatter diffraction. Ultramicroscopy, 111(8), 1395-1404. https://doi.org/10.1016/j.ultramic.2011.05.007

Britton, T. B., \& Wilkinson, A. J. (2012a). Stress fields and geometrically necessary dislocation density distributions near the head of a blocked slip band. Acta Materialia, 60(16), 5773-5782. https://doi.org/10.1016/j.actamat.2012.07.004

Britton, T. B., \& Wilkinson, A. J. (2012b). High resolution electron backscatter diffraction measurements of elastic strain variations in the presence of larger lattice rotations. Ultramicroscopy, 114, 82-95. https://doi.org/10.1016/j.ultramic.2012.01.004

Ceccato, A., Menegon, L., Pennacchioni, G., \& Morales, L. F. G. (2018). Myrmekite and strain weakening in granitoid mylonites. Solid Earth Discussions, 1-39. https://doi.org/10.5194/se-2018-70

Champness, P. E. (1987). Convergent beam electron diffraction. Mineralogical Magazine, 51, 33-48. https://doi.org/10.1180/ minmag.1987.051.359.04

Chen, K., Kunz, M., Li, Y., Zepeda-Alarcon, E., Sintubin, M., \& Wenk, H.-R. (2016). Compressional residual stress in Bastogne boudins revealed by synchrotron X-ray microdiffraction. Geophysical Research Letters, 43, 6178-6185. https://doi.org/10.1002/ $2016 \mathrm{gl069236}$

Chen, K., Kunz, M., Tamura, N.-R., \& Wenk, H. (2015). Residual stress preserved in quartz from the San Andreas Fault observatory at depth. Geology, 43, 219-222. https://doi.org/10.1130/g36443.1

Cross, A. J., Prior, D. J., Stipp, M., \& Kidder, S. (2017). The recrystallized grain size piezometer for quartz: An EBSD-based calibration. Geophysical Research Letters, 44, 6667-6674. https://doi.org/10.1002/2017gl073836

Detrez, F., Castelnau, O., Cordier, P., Merkel, S., \& Raterron, P. (2015). Effective viscoplastic behavior of polycrystalline aggregates lacking four independent slip systems inferred from homogenization methods; application to olivine. Journal of the Mechanics and Physics of Solids, 83, 199-220. https://doi.org/10.1016/j.jmps.2015.05.022

Dingley, D. J. (1984). Diffraction from sub-micron areas using electron backscattering in a scanning electron microscope. Scanning Electron Microscopy, 2, 569-575.

Dingley, D. J., Wilkinson, A. J., Meaden, G., \& Karamched, P. S. (2010). Elastic strain tensor measurement using electron backscatter diffraction in the SEM. Journal of Electron Microscopy, 59(Suppl 1), S155-S163. https://doi.org/10.1093/jmicro/dfq043

Dunne, F. P. E., Kiwanuka, R., \& Wilkinson, A. J. (2012). Crystal plasticity analysis of micro-deformation, lattice rotation and geometrically necessary dislocation density. Proceedings of the Royal Society A: Mathematical, Physical and Engineering Sciences, 468(2145), 2509-2531. https://doi.org/10.1098/rspa.2012.0050

Friedman, M. (1972). Residual elastic strain in rocks. Tectonophysics, 15, 297-330. https://doi.org/10.1016/0040-1951(72)90093-5

Gardner, J., Wheeler, J., Wallis, D., Hansen, L.N., \& Mariani, E. (2017). Mineral replacement reactions as a precursor to strain localisation: An (HR-)EBSD approach. American Geophysical Union, Fall Meeting 2017. MR41D-0439.

Halfpenny, A., Prior, D. J., \& Wheeler, J. (2006). Analysis of dynamic recrystallization and nucleation in a quartzite mylonite. Tectonophysics, 427(1-4), 3-14. https://doi.org/10.1016/j.tecto.2006.05.016

Hansen, L. N., Zimmerman, M. E., \& Kohlstedt, D. L. (2011). Grain boundary sliding in San Carlos olivine: Flow law parameters and crystallographic-preferred orientation. Journal of Geophysical Research, 116, B08201. https://doi.org/10.1029/2011jb008220

Heinisch, H. L. Jr., Sines, G., Goodman, J. W., \& Kirby, S. H. (1975). Elastic stresses and self-energies of dislocations of arbitrary orientation in anisotropic media: Olivine, orthopyroxene, calcite and quartz. Journal of Geophysical Research, 80(14), 1885-1896. https://doi.org/ 10.1029/JB080i014p01885

Holzhausen, G. R., \& Johnson, A. M. (1979). The concept of residual stress in rock. Tectonophysics, 58, 237-267. https://doi.org/10.1016/ 0040-1951(79)90311-1 
Humphreys, F. J., Huang, Y., Brough, I., \& Harris, C. (1999). Electron backscatter diffraction of grain and subgrain structures-Resolution considerations. Journal of Microscopy, 195(3), 212-216. https://doi.org/10.1046/j.1365-2818.1999.00579.x

Jiang, J., Britton, T. B., \& Wilkinson, A. J. (2013a). Measurement of geometrically necessary dislocation density with high resolution electron backscatter diffraction: Effects of detector binning and step size. Ultramicroscopy, 125, 1-9. https://doi.org/10.1016/j. ultramic.2012.11.003

Jiang, J., Britton, T. B., \& Wilkinson, A. J. (2013b). Mapping type III intragranular residual stress distributions in deformed copper polycrystals. Acta Materialia, 61(15), 5895-5904. https://doi.org/10.1016/j.actamat.2013.06.038

Jiang, J., Britton, T. B., \& Wilkinson, A. J. (2013c). Evolution of dislocation density distributions in copper during tensile deformation. Acta Materialia, 61(19), 7227-7239. https://doi.org/10.1016/j.actamat.2013.08.027

Jiang, J., Britton, T. B., \& Wilkinson, A. J. (2015). Evolution of intragranular stresses and dislocation densities during cyclic deformation of polycrystalline copper. Acta Materialia, 94, 193-204. https://doi.org/10.1016/j.actamat.2015.04.031

Jiang, J., Yang, J., Zhang, T., Dunne, F. P. E., \& Britton, T. B. (2015). On the mechanistic basis of fatigue crack nucleation in Ni superalloy containing inclusions using high resolution electron backscatter diffraction. Acta Materialia, 97, 367-379. https://doi.org/10.1016/j. actamat.2015.06.035

Jiang, J., Zhang, T., Dunne, F. P. E., \& Britton, T. B. (2016). Deformation compatibility in a single crystalline Ni superalloy. Proceedings of the Royal Society A: Mathematical, Physical and Engineering Science, 472(2185), 20150690. https://doi.org/10.1098/rspa.2015.0690

Karamched, P. S., \& Wilkinson, A. J. (2011). High resolution electron back-scatter diffraction analysis of thermally and mechanically induced strains near carbide inclusions in a superalloy. Acta Materialia, 59(1), 263-272. https://doi.org/10.1016/j. actamat.2010.09.030

Kartal, M. E., Kiwanuka, R., \& Dunne, F. P. E. (2015). Determination of sub-surface stresses at inclusion in single crystal superalloy using HR-EBSD, crystal plasticity and inverse eigenstrain analysis. International Journal of Solids and Structures, 67-68, 27-39. https://doi.org/ 10.1016/j.ijsolstr.2015.02.023

Kohn, M. J. (2014). "Thermoba-Raman-try": Calibration of spectroscopic barometers and thermometers for mineral inclusions. Earth and Planetary Science Letters, 388, 187-196. https://doi.org/10.1016/j.epsl.2013.11.054

Kröner, E. (1958). Continuum theory of dislocations and self-stresses. Ergebnisse Der Angewandten Mathematik, 5, 1327-1347.

Kruhl, J. H. (1996). Prism- and basal-plane parallel subgrain boundaries in quartz: A microstructural geothermobarometer. Journal of Metamorphic Geology, 14, 581-589.

Kumamoto, K. M., Thom, C. A., Wallis, D., Hansen, L. N., Armstrong, D. E. J., Warren, J. M., et al. (2017). Size effects resolve discrepancies in 40 years of work on low-temperature plasticity in olivine. Science Advances, 3(9), e1701338. https://doi.org/10.1126/sciadv.1701338

Linker, M. F., Kirby, S. H., Ord, A., \& Christie, J. M. (1984). Effects of compression direction on the plasticity and rheology of hydrolytically weakened synthetic quartz crystals at atmospheric pressure. Journal of Geophysical Research, 89(B6), 4241-4255. https://doi.org/ 10.1029/jb089ib06p04241

Littlewood, P. D., Britton, T. B., \& Wilkinson, A. J. (2011). Geometrically necessary dislocation density distributions in Ti-6Al-4V deformed in tension. Acta Materialia, 59(16), 6489-6500. https://doi.org/10.1016/j.actamat.2011.07.016

Lloyd, G. E. (1987). Atomic number and crystallographic contrast images with the SEM: A review of backscattered electron techniques. Mineralogical Magazine, 51(359), 3-19. https://doi.org/10.1180/minmag.1987.051.359.02

Lloyd, G. E. (2004). Microstructural evolution in a mylonitic quartz simple shear zone: The significant roles of dauphine twinning and misorientation. Geological Society, London, Special Publications, 224(1), 39-61. https://doi.org/10.1144/gsl.sp.2004.224.01.04

Lloyd, G. E., Farmer, A. B., \& Mainprice, D. (1997). Misorientation analysis and the formation and orientation of subgrain and grain boundaries. Tectonophysics, 279(1-4), 55-78. https://doi.org/10.1016/s0040-1951(97)00115-7

Mainprice, D., Bascou, J., Cordier, P., \& Tommasi, A. (2004). Crystal preferred orientations of garnet: Comparison between numerica simulations and electron back-scattered diffraction (EBSD) measurements in naturally deformed eclogites. Journal of Structural Geology, 26(11), 2089-2102. https://doi.org/10.1016/j.jsg.2004.04.008

Mainprice, D., Bouchez, J.-L., Blumenfeld, P., \& Tubià, J. M. (1986). Dominant c slip in naturally deformed quartz: Implications for dramatic plastic softening at high temperature. Geology, 14, 819-822. https://doi.org/10.1130/0091-7613(1986)14<819:dcsind >2.0.co;2

Maurice, C., Driver, J. H., \& Fortunier, R. (2012). On solving the orientation gradient dependency of high angular resolution EBSD. Ultramicroscopy, 113, 171-181. https://doi.org/10.1016/j.ultramic.2011.10.013

Maurice, C., Dzieciol, K., \& Fortunier, R. (2011). A method for accurate localisation of EBSD pattern centres. Ultramicroscopy, 111(2), 140-148. https://doi.org/10.1016/j.ultramic.2010.10.007

Maurice, C., \& Fortunier, R. (2008). A 3D Hough transform for indexing EBSD and Kossel patterns. Journal of Microscopy, 230(3), 520-529. https://doi.org/10.1111/j.1365-2818.2008.02045.x

Maurice, C., Quey, R., Fortunier, R., \& Driver, J. H. (2013). High angular resolution EBSD and its materials applications. Microstructural Design of Advanced Engineering Materials, 339-365. https://doi.org/10.1002/9783527652815.ch14

Mesarovic, S. D., Forest, S., \& Jaric, J. P. (2015). Size-dependent energy in crystal plasticity and continuum dislocation models. Proceedings of the Royal Society A, 471(2175), 20140868. https://doi.org/10.1098/rspa.2014.0868

Mikami, Y., Oda, K., Kamaya, M., \& Mochizuki, M. (2015). Effect of reference point selection on microscopic stress measurement using EBSD. Materials Science and Engineering A, 647, 256-264. https://doi.org/10.1016/j.msea.2015.09.004

Morales, L. F. G., Lloyd, G. E., \& Mainprice, D. (2014). Fabric transitions in quartz via viscoplastic self-consistent modeling part I: Axial compression and simple shear under constant strain. Tectonophysics, 636, 52-69. https://doi.org/10.1016/j.tecto.2014.08.011

Nolze, G. (2007). Image distortions in SEM and their influences on EBSD measurements. Ultramicroscopy, 107(2-3), 172-183. https://doi. org/10.1016/j.ultramic.2006.07.003

van Noort, R., Wolterbeek, T., Drury, M., Kandianis, M., \& Spiers, C. (2017). The force of crystallization and fracture propagation during insitu carbonation of peridotite. Minerals, 7(10), 190. https://doi.org/10.3390/min7100190

Nye, J. F. (1953). Some geometrical relations in dislocated crystals. Acta Metallurgica, 1(2), 153-162. https://doi.org/10.1016/00016160(53)90054-6

Okudaira, T., Takeshita, T., Hara, I., \& Ando, J.-I. (1995). A new estimate of the conditions for transition from basal $<a>$ to prism [c] slip in naturally deformed quartz. Tectonophysics, 250(1-3), 31-46. https://doi.org/10.1016/0040-1951(95)00039-4

Pagliaro, P., Prime, M. B., Robinson, J. S., Clausen, B., Swenson, H., Steinzig, M., \& Zuccarello, B. (2011). Measuring inaccessible residual stresses using multiple methods and superposition. Experimental Mechanics, 51(7), 1123-1134. https://doi.org/10.1007/s11340-0109424-5

Pantleon, W. (2008). Resolving the geometrically necessary dislocation content by conventional electron backscattering diffraction. Scripta Materialia, 58(11), 994-997. https://doi.org/10.1016/j.scriptamat.2008.01.050 
Parsons, A. J., Law, R. D., Lloyd, G. E., Phillips, R. J., \& Searle, M. P. (2016). Thermo-kinematic evolution of the Annapurna-Dhaulagiri Himalaya, central Nepal: The Composite Orogenic System. Geochemistry, Geophysics, Geosystems, 17, 1511-1539. https://doi.org/ $10.1002 / 2015 \mathrm{gc006184}$

Plancher, E., Petit, J., Maurice, C., Favier, V., Saintoyant, L., Loisnard, D., et al. (2015). On the Accuracy of elastic strain field measurements by Laue microdiffraction and high-resolution EBSD: A cross-validation experiment. Experimental Mechanics, 56(3), 483-492. https:// doi.org/10.1007/s11340-015-0114-1

Poirier, J. P., 1982. On transformation plasticity. Journal of Geophysical Research, 87, 6791-6797. https://doi.org/10.1029/jb087ib08p06791 Popov, V. L., \& Kröner, E. (2001). Theory of elastoplastic media with mesostructured. Theoretical and Applied Fracture Mechanics, 37(1-3), 299-310. https://doi.org/10.1016/S0167-8442(01)00086-6

Prior, D. J. (1999). Problems in determining the misorientation axes, for small angular misorientations, using electron backscatter diffraction in the SEM. Journal of Microscopy, 195(3), 217-225. https://doi.org/10.1046/j.1365-2818.1999.00572.x

Prior, D. J., Boyle, A. P., Brenker, F., Cheadle, M. C., Day, A., Lopez, G., et al. (1999). The application of electron backscatter diffraction and orientation contrast imaging in the SEM to textural problems in rocks. The American Mineralogist, 84(11-12), 1741-1759. https://doi.org/ 10.2138/am-1999-11-1204

Prior, D. J., Mariani, E., \& Wheeler, J. (2009). EBSD in the Earth sciences: Applications, common practice, and challenges. Electron Backscatter Diffraction in Materials Science, 345-360. https://doi.org/10.1007/978-0-387-88136-2_26

Prior, D. J., Wheeler, J., Peruzzo, L., Spiess, R., \& Storey, C. (2002). Some garnet microstructures: An illustration of the potential of orientation maps and misorientation analysis in microstructural studies. Journal of Structural Geology, 24(6-7), 999-1011. https://doi. org/10.1016/s0191-8141(01)00087-6

Qi, C., Hansen, L. N., Wallis, D., Holtzman, B. K., \& Kohlstedt, D. L. (2018). Crystallographic preferred orientation of olivine in sheared partially molten rocks: The source of the "a-c switch". Geochemistry, Geophysics, Geosystems, 19(2), 316-336. https://doi.org/10.1002/ $2017 \mathrm{gc} 007309$

Rosenfeld, J. L., \& Chase, A. B. (1961). Pressure and temperature of crystallization from elastic effects around solid inclusions in minerals? American Journal of Science, 259(7), 519-541. https://doi.org/10.2475/ajs.259.7.519

Ruggles, T. J., Bomarito, G. F., Qiu, R. L., \& Hochhalter, J. D. (2018). New levels of high angular resolution EBSD performance via inverse compositional Gauss-Newton based digital image correlation. Ultramicroscopy, 195, 85-92. https://doi.org/10.1016/j. ultramic.2018.08.020

Ruggles, T. J., Fullwood, D. T., \& Kysar, J. W. (2016). Resolving geometrically necessary dislocation density onto individual dislocation types using EBSD-based continuum dislocation microscopy. International Journal of Plasticity, 76, 231-243. https://doi.org/10.1016/j. ijplas.2015.08.005

Ruggles, T. J., Rampton, T. M., Khosravani, A., \& Fullwood, D. T. (2016). The effect of length scale on the determination of geometrically necessary dislocations via EBSD continuum dislocation microscopy. Ultramicroscopy, 164, 1-10. https://doi.org/10.1016/j. ultramic.2016.03.003

Schäffer, A.-K., Jäpel, T., Zaefferer, S., Abart, R., \& Rhede, D. (2014). Lattice strain across Na-K interdiffusion fronts in alkali feldspar: An electron back-scatter diffraction study. Physics and Chemistry of Minerals, 41(10), 795-804. https://doi.org/10.1007/s00269-0140692-y

Speller, S. C., Dudin, P., Fitzgerald, S., Hughes, G. M., Kruska, K., Britton, T. B., et al. (2014). High-resolution characterization of microstructural evolution inRbxFe2-ySe2crystals on annealing. Physical Review B: Condensed Matter and Materials Physics, $90(2), 024520$. https://doi.org/10.1103/physrevb.90.024520

Sun, C. T., \& Jin, Z.-H. (2012). The elastic stress field around a crack tip. Fracture Mechanics of Ceramics, 25-75. https://doi.org/10.1016/ b978-0-12-385001-0.00003-1

Sun, S., Adams, B. L., \& King, W. E. (2000). Observations of lattice curvature near the interface of a deformed aluminium bicrystal. Philosophical Magazine A, 80(1), 9-25. https://doi.org/10.1080/01418610008212038

Tasaka, M., Zimmerman, M. E., Kohlstedt, D. L., Stünitz, H., \& Heilbronner, R. (2017). Rheological weakening of olivine orthopyroxene aggregates due to phase mixing: Part 2. Microstructural development. Journal of Geophysical Research: Solid Earth, 122, 7597-7612. https://doi.org/10.1002/2017jb014311

Tommasi, A., Langone, A., Padrón-Navarta, J. A., Zanetti, A., \& Vauchez, A. (2017). Hydrous melts weaken the mantle, crystallization of pargasite and phlogopite does not: Insights from a petrostructural study of the Finero peridotites, southern Alps. Earth and Planetary Science Letters, 477, 59-72. https://doi.org/10.1016/j.epsl.2017.08.015

Tong, V., Jiang, J., Wilkinson, A. J., \& Britton, T. B. (2015). The effect of pattern overlap on the accuracy of high resolution electron backscatter diffraction measurements. Ultramicroscopy, 155, 62-73. https://doi.org/10.1016/j.ultramic.2015.04.019

Trepmann, C. A., \& Stöckhert, B. (2003). Quartz microstructures developed during non-steady state plastic flow at rapidly decaying stress and strain rate. Journal of Structural Geology, 25(12), 2035-2051. https://doi.org/10.1016/s0191-8141(03)00073-7

Trimby, P. W., Prior, D. J., \& Wheeler, J. (1998). Grain boundary hierarchy development in a quartz mylonite. Journal of Structural Geology, 20(7), 917-935. https://doi.org/10.1016/s0191-8141(98)00026-1

Troost, K. Z., van der Sluis, P., \& Gravesteijn, D. J. (1993). Microscale elastic-strain determination by backscatter Kikuchi diffraction in the scanning electron microscope. Applied Physics Letters, 62(10), 1110-1112. https://doi.org/10.1063/1.108758

Vermeij, T., de Graef, M., \& Hoefnagels, J. (2019). Demonstrating the potential of accurate absolute cross-grain stress and orientation correlation using electron backscatter diffraction. Scripta Materialia, 162, 266-271. https://doi.org/10.1016/j.scriptamat.2018.11.030

Vermeij, T., \& Hoefnagels, J. P. M. (2018). A consistent full-field integrated DIC framework for HR-EBSD. Ultramicroscopy, 191, 44-50. https://doi.org/10.1016/j.ultramic.2018.05.001

Vilalta-Clemente, A., Naresh-Kumar, G., Nouf-Allehiani, M., Gamarra, P., di Forte-Poisson, M. A., Trager-Cowan, C., \& Wilkinson, A. J. (2017). Cross-correlation based high resolution electron backscatter diffraction and electron channelling contrast imaging for strain mapping and dislocation distributions in InAlN thin films. Acta Materialia, 125, 125-135. https://doi.org/10.1016/j.actamat.2016.11.039

Villanova, J., Maurice, C., Micha, J.-S., Bleuet, P., Sicardy, O., \& Fortunier, R. (2012). Multiscale measurements of residual strains in a stabilized zirconia layer. Journal of Applied Crystallography, 45(5), 926-935. https://doi.org/10.1107/s0021889812028427

Villert, S., Maurice, C., Wyon, C., \& Fortunier, R. (2009). Accuracy assessment of elastic strain measurement by EBSD. Journal of Microscopy, 233(2), 290-301. https://doi.org/10.1111/j.1365-2818.2009.03120.x

Wallis, D., Hansen, L. N., Britton, T. B., \& Wilkinson, A. J. (2016). Geometrically necessary dislocation densities in olivine obtained using high-angular resolution electron backscatter diffraction. Ultramicroscopy, 168, 34-45. https://doi.org/10.1016/j.ultramic.2016.06.002

Wallis, D., Hansen, L. N., Britton, T. B., \& Wilkinson, A. J. (2017). Dislocation interactions in olivine revealed by HR-EBSD. Journal of Geophysical Research: Solid Earth, 122, 7659-7678. https://doi.org/10.1002/2017jb014513 
Wallis, D., Lloyd, G. E., \& Hansen, L. N. (2018). The role of strain hardening in the transition from dislocation-mediated to frictional deformation of marbles within the Karakoram Fault Zone, NW India. Journal of Structural Geology, 107, 25-37. https://doi.org/10.1016/ j.jsg.2017.11.008

Wallis, D., Parsons, A. J., \& Hansen, L. N. (2017). Quantifying geometrically necessary dislocations in quartz using HR-EBSD: Application to chessboard subgrain boundaries. Journal of Structural Geology. https://doi.org/10.1016/j.jsg.2017.12.012

Weikusat, I., Kuiper, E.-J. N., Pennock, G. M., Kipfstuhl, S., \& Drury, M. R. (2017). EBSD analysis of subgrain boundaries and dislocation slip systems in Antarctic and Greenland ice. Solid Earth, 8(5), 883-898. https://doi.org/10.5194/se-8-883-2017

Wheeler, J., Mariani, E., Piazolo, S., Prior, D. J., Trimby, P., \& Drury, M. R. (2009). The weighted Burgers vector: A new quantity for constraining dislocation densities and types using electron backscatter diffraction on 2D sections through crystalline materials. Journal of Microscopy, 233(3), 482-494. https://doi.org/10.1111/j.1365-2818.2009.03136.x

Wilkinson, A. J. (1996). Measurement of elastic strains and small lattice rotations using electron back scatter diffraction. Ultramicroscopy, 62(4), 237-247. https://doi.org/10.1016/0304-3991(95)00152-2

Wilkinson, A. J. (2000). Advances in SEM-based diffraction studies of defects and strains in semiconductors. Journal of Electron Microscopy, 49(2), 299-310. https://doi.org/10.1093/oxfordjournals.jmicro.a023810

Wilkinson, A. J. (2001). A new method for determining small misorientations from electron back scatter diffraction patterns. Scripta Materialia, 44(10), 2379-2385. https://doi.org/10.1016/s1359-6462(01)00943-5

Wilkinson, A. J., \& Britton, T. B. (2012). Strains, planes, and EBSD in materials science. Materials Today, 15(9), 366-376. https://doi.org/ $10.1016 / \mathrm{s} 1369-7021(12) 70163-3$

Wilkinson, A. J., \& Hirsch, P. B. (1997). Electron diffraction based techniques in scanning electron microscopy of bulk materials. Micron, 28(4), 279-308. https://doi.org/10.1016/s0968-4328(97)00032-2

Wilkinson, A. J., Meaden, G., \& Dingley, D. J. (2006a). High-resolution elastic strain measurement from electron backscatter diffraction patterns: New levels of sensitivity. Ultramicroscopy, 106(4-5), 307-313. https://doi.org/10.1016/j.ultramic.2005.10.001

Wilkinson, A. J., Meaden, G., \& Dingley, D. J. (2006b). High resolution mapping of strains and rotations using electron backscatter diffraction. Materials Science and Technology, 22(11), 1271-1278. https://doi.org/10.1179/174328406x130966

Wilkinson, A. J., \& Randman, D. (2010). Determination of elastic strain fields and geometrically necessary dislocation distributions near nanoindents using electron back scatter diffraction. Philosophical Magazine, 90(9), 1159-1177. https://doi.org/10.1080/ 14786430903304145

Wilkinson, A. J., Tarleton, E., Vilalta-Clemente, A., Jiang, J., Britton, T. B., \& Collins, D. M. (2014). Measurement of probability distributions for internal stresses in dislocated crystals. Applied Physics Letters, 105(18), 181907. https://doi.org/10.1063/1.4901219

Wright, S. I., \& Adams, B. L. (1992). Automatic analysis of electron backscatter diffraction patterns. Metallurgical Transactions A, 23(3), 759-767. https://doi.org/10.1007/bf02675553

Zhang, T., Jiang, J., Shollock, B. A., Britton, T. B., \& Dunne, F. P. E. (2015). Slip localization and fatigue crack nucleation near a nonmetallic inclusion in polycrystalline nickel-based superalloy. Materials Science and Engineering A, 641, 328-339. https://doi.org/ 10.1016/j.msea.2015.06.070

Zheng, Z., Prastiti, N. G., Balint, D. S., \& Dunne, F. P. E. (2019). The dislocation configurational energy density in discrete dislocation plasticity. Journal of the Mechanics and Physics of Solids, 129, 39-60. https://doi.org/10.1016/j.jmps.2019.04.015 